\title{
An in vitro batch fermentation protocol for studying the contribution of food to gut microbiota composition and functionality
}

\author{
Sergio Pérez-Burillo ${ }^{1}$, Silvia Molino ${ }^{1}$, Beatriz Navajas-Porras ${ }^{1}$, Álvaro Jesús Valverde-Moya ${ }^{1}$, \\ Daniel Hinojosa-Nogueira $\mathbb{D}^{1}$, Alicia López-Maldonado ${ }^{1}$, Silvia Pastoriza $\mathbb{D}^{1}$ and \\ José Ángel Rufián-Henares $\mathbb{1}^{1,2}$ 约
}

\begin{abstract}
Knowledge of the effect of foods on gut microbiota composition and functionality is expanding. To isolate the effect of single foods and/or single nutrients (i.e., fiber, polyphenols), this protocol describes an in vitro batch fermentation procedure to be carried out after an in vitro gastrointestinal digestion. Therefore, this is an extension of the previous protocol described by Brodkorb et al. (2019) for studying in vitro digestion. The current protocol uses an oligotrophic fermentation medium with peptone and a high concentration of fecal inoculum from human fecal samples both to provide the microbiota and as the main source of nutrients for the bacteria. This protocol is recommended for screening work to be performed when many food samples are to be studied. It has been used successfully to study gut microbiota fermentation of different foodstuffs, giving insights into their functionality, community structure or ability to degrade particular substances, which can contribute to the development of personalized nutrition strategies. The procedure does not require a specific level of expertise. The protocol takes 4-6 $\mathrm{h}$ for preparation of fermentation tubes and $20 \mathrm{~h}$ for incubation.
\end{abstract}

This protocol is an extension to: Nat. Protoc. 14, 991-1014 (2019): https://doi.org/10.1038/s41596-018-0119-1

Gut microbiota have recently become a major focus in the study of human health. Gut microbes are closely related to human health ${ }^{1}$ and have been linked to important conditions such as inflammatory bowel disease, immune system disorders, obesity or even autism spectrum disorders ${ }^{2}$. Gut microbiota can be disturbed by many different factors such as age, antibiotics and exercise, but diet, and specifically food components, are most probably the main drivers causing changes in gut microbiota behavior $^{3}$. It has been demonstrated extensively how the gut of human populations with different dietary patterns is colonized by different microbial communities, which in turn reflects on people's health $^{4-6}$. Therefore, many efforts have been put into developing different strategies to study the gut microbial community and its functionality, such as in vitro models (including static batch fermentations and continuous systems), animal models and human clinical/observational studies ${ }^{7}$. Each one of them has its own limitations and advantages/disadvantages. Although human studies provide the information with the highest physiological relevance, in vitro models are still essential to test specific foods or food components and for initial screenings ${ }^{7}$.

Development of the protocol

There are essentially two types of in vitro fermentation models for studying gut microbiota: those based on batch fermentations and those based on continuous systems. The latter are closer to physiological conditions than batch fermentations ${ }^{7}$. Moreover, they allow a better representation of the gut microbial communities; they can be kept stable for longer periods of time, even several weeks, and mimic the conditions of the different portions of the colon in an automatized manner ${ }^{7}$. A widely used continuous system is the Simulator of the Human Intestinal Microbial Ecosystem (SHIME) ${ }^{8}$. This is a complex and expensive system comprising five stages, two of which mimic gastrointestinal digestion and three of which mimic colonic fermentation. There are also continuous systems that are

'Departamento de Nutrición y Bromatología, Instituto de Nutrición y Tecnología de Alimentos, Centro de Investigación Biomédica, Universidad de Granada, Granada, Spain. ${ }^{2}$ Instituto de Investigación Biosanitaria ibs.GRANADA, Universidad de Granada, Granada, Spain. ${ }^{凶}$ e-mail: jarufian@ugr.es 
less complicated but still composed of three vessels to mimic colonic conditions and automated to control $\mathrm{pH}$ and to pump the contents from one vessel to another ${ }^{9}$. These are large systems that need their own room and specialized operators, which in turn make them sometimes inappropriate for certain laboratories. Miniaturized systems have been developed to overcome this problem ${ }^{10}$. Regardless of their size or complexity, they all work in a similar way and try to mimic the different portions of the colon, controlling $\mathrm{pH}$ and nutrients. Their main disadvantage is that only one sample can be studied at once, and the experiment usually takes at least a month since they first stabilize microbial communities for 1-2 weeks and then add the component subject to study and keep the experiment running for another 1-2 weeks'.

On the other hand, batch fermentations, generally carried out in test tubes, enable many samples to be studied at once and within a short period of time, usually $24-48 \mathrm{~h}^{11,12}$. Therefore, these models become convenient when the objective is to make an initial screening of many foodstuffs or food components ${ }^{13}$. However, they are less physiologically relevant, and bacterial waste products will eventually accumulate, hence the need for a shorter experimental duration ${ }^{7}$. Still, both in vitro approaches could complement each other, using batch fermentations for initial screening of foods or food components and continuous systems for the selected ones, according to the investigator's needs.

Because many different in vitro batch fermentations have been used, there is a need to propose a common methodology, since a variation in the conditions (e.g., fecal material origin, fecal slurry concentration, incubation time, culture medium composition) would affect microbial communities and their metabolism (Table 1). The first issue that arises is whether to perform a prior in vitro digestion; as Table 1 shows, some researchers perform in vitro digestion, while others do not. This will translate into the presence or absence of enzymes during colonic fermentation (they are proteins, so they can be used by microbes) and presence or absence of bile salts (which are also transformed by microbes). In addition, in the case of actual foodstuffs, in vitro digestion would break down their structure, making nutrients more accessible for microbes, which will not be achieved if prior digestion has not been performed. Additionally, when a previous digestion is performed, it is carried out using different protocols across experiments, which translates into different amounts of digestion components available for colonic fermentation. Another main difference that can be observed in Table 1 is the fermentation medium. There are two main types-a rich nutrient medium or a minimum medium. A rich medium could be more appropriate for continuous systems since these aim to stabilize the microbial community before adding the substance under study. For batch fermentations, a minimum medium can help highlight the metabolism of the substance under study and quickly identify the resulting metabolites or involved bacteria, with results that are easier to interpret. Afterward, substances selected by the investigator could be tested in a continuous system with a rich medium over several weeks. Moreover, even among those who use the same type of medium, concentrations as well as nutrients usually vary between experiments (Table 1). In fact, Mould et al. proposed a simplified medium composed only of a buffering solution, cysteine, a sulfur source and several minerals ${ }^{14}$. Finally, fecal material source and fecal slurry concentration vary across experiments, which will affect the results.

Therefore, a need for standardized conditions has been identified, and the authors propose an in vitro protocol to simulate colonic fermentation based on batch culture and using human feces as the source of gut microbes coupled with a prior in vitro digestion. This prior phase will not be discussed here since the authors propose the use of the INFOGEST digestion protocol already published in this journal ${ }^{15}$. The proposed in vitro digestion-fermentation protocol has been successfully used by the authors to test antioxidant capacity after fermenting chicken, whole grain bread, lentils, orange, tomato, yogurt and peanuts ${ }^{16}$; and to study how microbial communities and their functionality are affected by chicken, bread, pepper, chickpeas, banana subjected to different cooking methods ${ }^{17}$, roasted and green coffee $^{18}$, salami with different potential prebiotic agents added ${ }^{19}$, mannooligosaccharides extracted from spent coffee grounds ${ }^{20}$, and melanoidins extracted from different food sources (coffee, black and pilsner beer, breakfast cereal, bread crust, biscuits, chocolate, balsamic vinegar and sweet wine $)^{21}$. Other authors also used this protocol to study the modulatory effect of polyphenols and sesquiterpene lactones from artichoke heads ${ }^{22}$ or Chlorella spp. ${ }^{23}$ on gut microbiota composition and functionality.

\section{Overview of the procedure}

The procedure is summarized in Fig. 1. It can be divided into seven basic stages (see 'Experimental design' for further information): (i) fecal material collection, (ii) preparation of the equipment and 


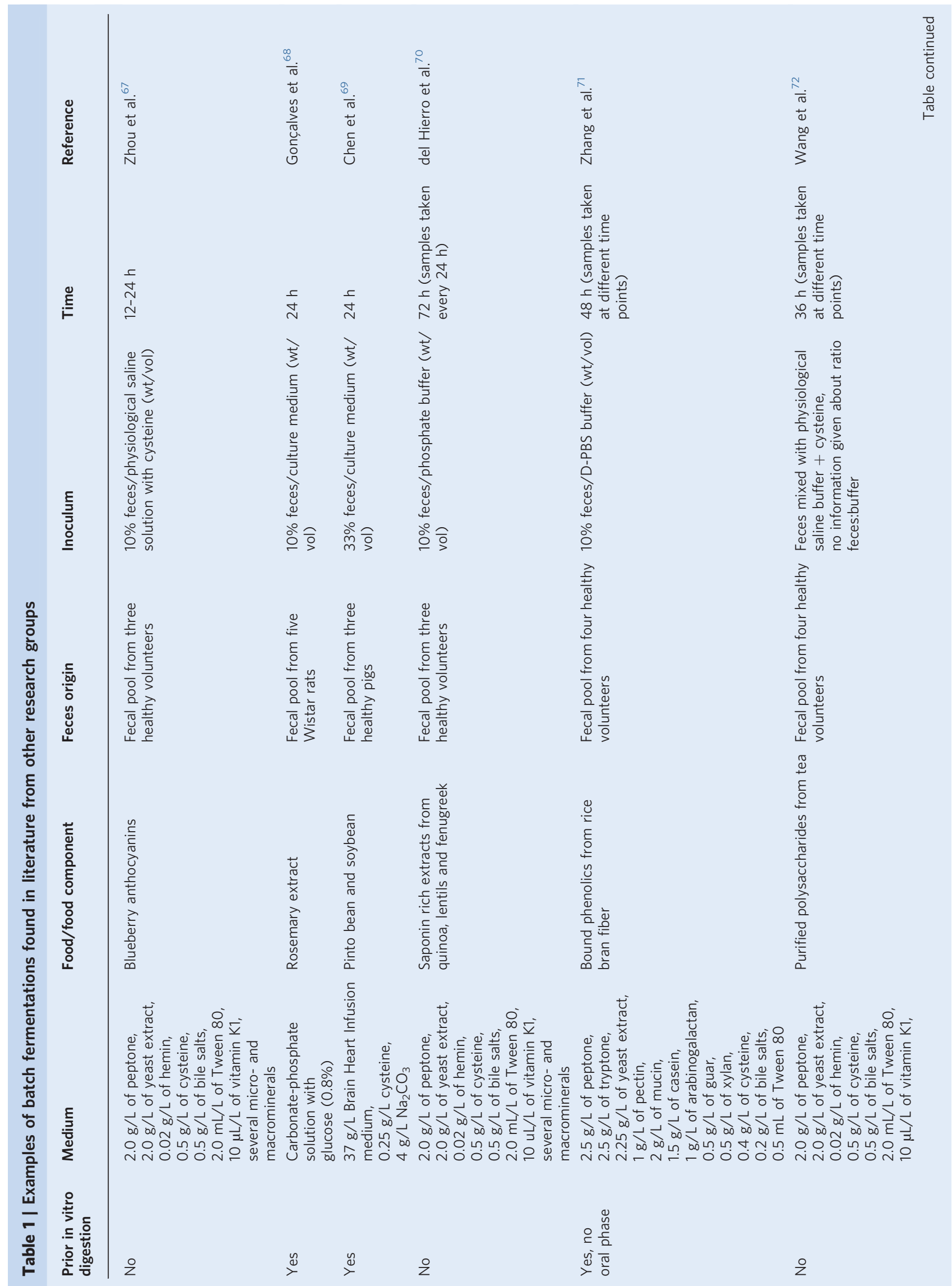




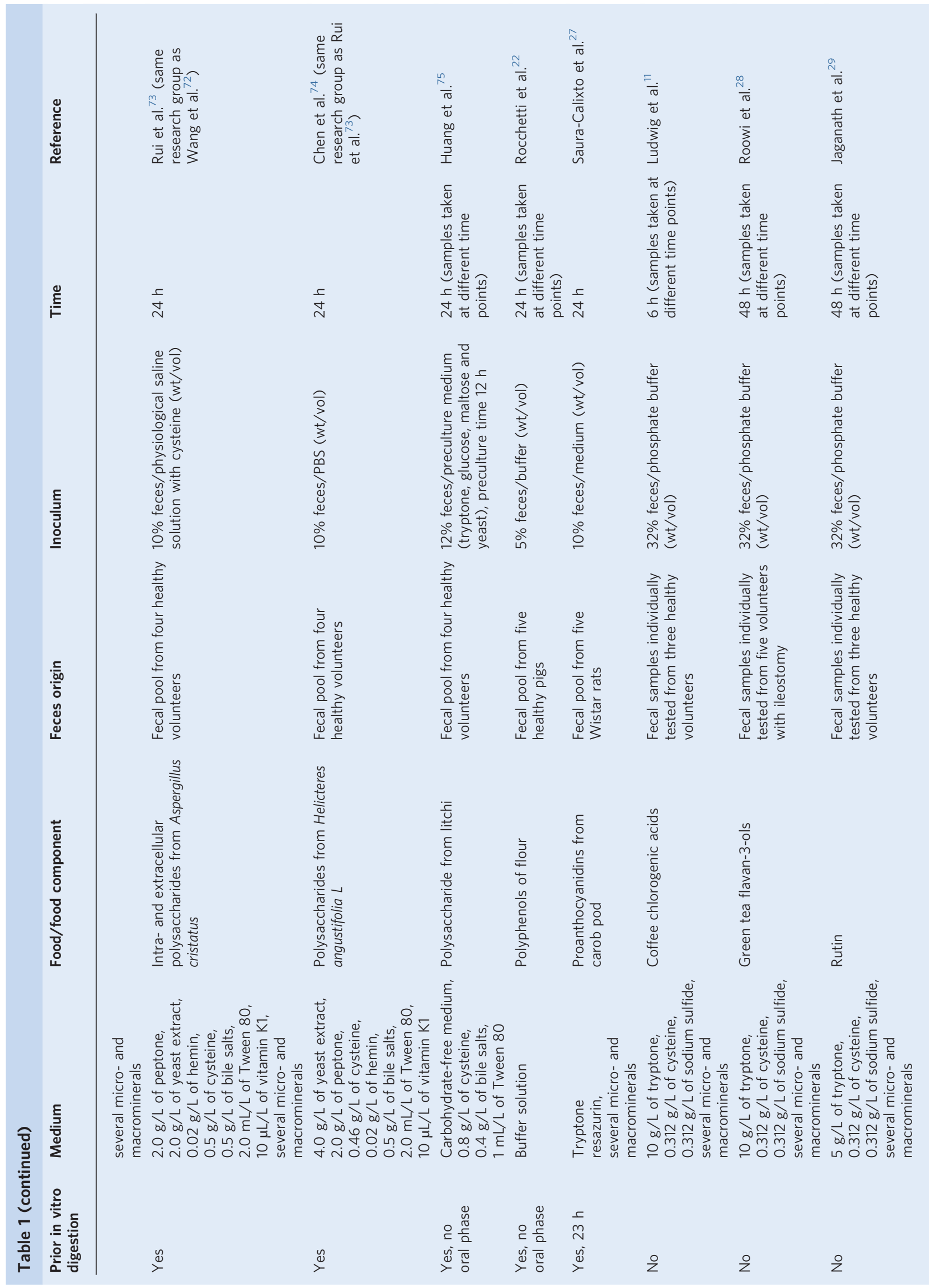




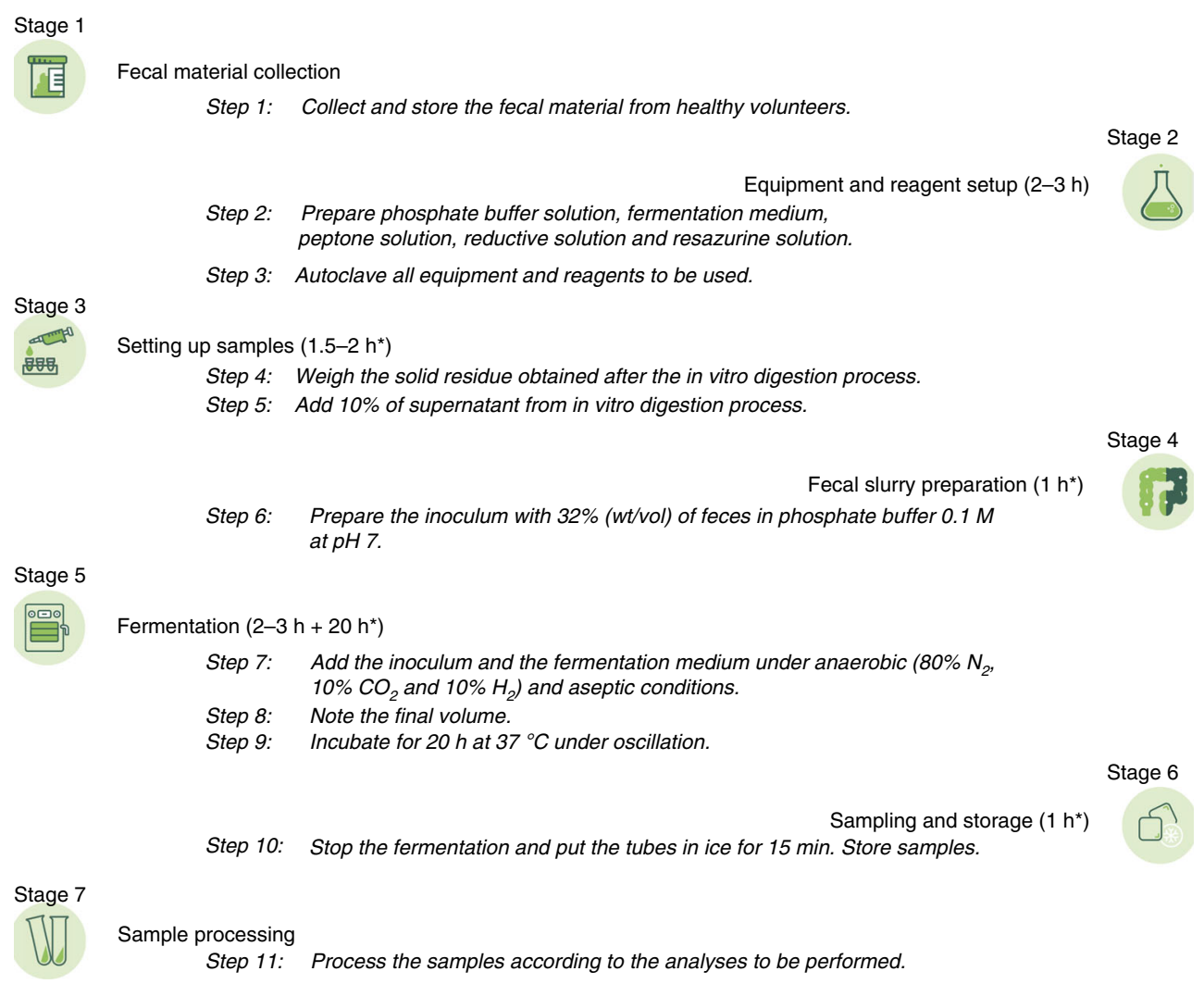

Fig. 1 | In vitro fermentation process. ${ }^{\star}$ The time estimated to process $\sim 50$ samples.

reagents, (iii) setup of the in vitro digestion samples to be fermented, (iv) preparation of the fecal slurry, (v) fermentation, (vi) sampling and (vii) sample processing.

Fecal material should be collected from volunteers (always at least four to pool together the feces and minimize interindividual variation ${ }^{5}$ ) under conditions dependent on the aim of the experiments; if the aim is to investigate regular microbiota, feces should come from healthy individuals (e.g., not taking antibiotics, having a normal body mass index); however, if the aim is to investigate microbiota from a particular illness, then the feces should be collected from volunteers with that condition. Specimens should always be collected in sterile containers specifically made for that purpose, and gloves should be worn to avoid contamination. Fecal material should ideally be collected the same morning when the experiment is going to be carried out; however, fecal material can be stored at $4{ }^{\circ} \mathrm{C}$ for $24 \mathrm{~h}$, or kept stable for 2 months frozen at $-80{ }^{\circ} \mathrm{C}$ with glycerol to protect microbes from ice crystals.

Preparation of the materials and reagents involves firstly the preparation of the fermentation medium with peptone and resazurin and oxygen removal by bubbling nitrogen $(\mathrm{N})$ through it, followed by autoclaving. Cysteine and sodium sulfide (reductive solution) are added afterward to avoid losing cysteine during the thermal treatment. Phosphate buffer for fecal slurry preparation is also made and autoclaved. Materials to be used during the experiment are also autoclaved: Milli-Q water, pipette tips and laboratory spoons.

The fecal slurry is prepared at $32 \%$ feces (wt/vol) in phosphate buffer. Each fermentation tube carries $7.5 \mathrm{~mL}$ of medium, $2 \mathrm{~mL}$ of fecal slurry and $0.5 \mathrm{~g}$ of substrate sample from in vitro digestion. Tubes are kept at $37^{\circ} \mathrm{C}$ with oscillating shaking at $20 \mathrm{rpm}$ for $20 \mathrm{~h}$. Right after, microbial activity is stopped by placing the tubes on ice (see 'Experimental conditions'). A typical fermentation example with quantities and volumes added is described in Table 2.

Sampling and sample storage will vary depending on the analysis to be performed. Therefore, an appropriate sampling strategy should be considered before carrying out the experiment (see 'Experimental design' (Stage 6) and Table 3 for details). Finally, samples are processed for assessesment of substrate degradation, for metabolomics analysis (to measure the presence of certain metabolites and study gut microbial functionality) or for $16 \mathrm{~S}$ RNA sequencing or shotgun metagenomics analysis to reveal gut microbial community structure. 
Table 2 | Example of an in vitro fermentation reaction setup

Solid residue, $\mathrm{g}$

In vitro digestion

In vitro fermentation

3

Component
Digestion solid residue/chemical of interest, $\mathrm{g}$ $10 \%$ of digestion supernatant, $\mathrm{mL}$ Inoculum (32\% wt/vol; feces/phosphate buffer $\mathrm{pH} 7), \mathrm{mL}$

Medium (peptone $15 \mathrm{~g} / \mathrm{L}+50 \mathrm{~mL}$ of reductive solution/L of peptone), $\mathrm{mL}$

Bubble nitrogen

Incubation
Supernatant volume, $\mathrm{mL}$

$\begin{aligned} & 37 \\ & \text { Quantity } \\ & 0.5 \\ & 0.5 \times 3.7 / 3=0.62 \\ & 2\end{aligned}$
7.5
1 min
Oscillation at $20 \mathrm{rpm}, 20 \mathrm{~h}$,
$37^{\circ} \mathrm{C}$

Table 3 | Sampling timing and conditions

\begin{tabular}{|c|c|c|c|}
\hline Application & Objective & $\begin{array}{l}\text { Samples to take, measurements to } \\
\text { make and timing }\end{array}$ & Sampling procedure \\
\hline Metabolomics & $\begin{array}{l}\text { Measurement of different } \\
\text { metabolites }\end{array}$ & $\begin{array}{l}\text { Sample fecal inoculum }{ }^{\text {a }} \text {, weigh blank } \\
\text { tube before incubation }{ }^{a} \text {, weigh sample } \\
\text { tube before incubation }{ }^{a} \text {, weigh blank } \\
\text { tube after incubation }{ }^{a} \text {, weigh sample } \\
\text { tube after incubation }{ }^{a} \text {, take samples } \\
\text { every } X \text { hours during incubation }\end{array}$ & $\begin{array}{l}\text { Pipette } 1 \mathrm{~mL} \text { for each analysis foreseen, } \\
\text { and store at }-80^{\circ} \mathrm{C}\end{array}$ \\
\hline $\begin{array}{l}16 \mathrm{~S} \text { rRNA amplicon } \\
\text { sequencing or shotgun } \\
\text { metagenomics }\end{array}$ & $\begin{array}{l}\text { Explore gut microbial } \\
\text { community structure }\end{array}$ & $\begin{array}{l}\text { Sample fecal inoculum }{ }^{\text {a }} \text {, weigh blank } \\
\text { tube after incubation }{ }^{a} \text {, weigh sample } \\
\text { tube after incubation }{ }^{a} \text {, take samples } \\
\text { every } X \text { hours during incubation }\end{array}$ & $\begin{array}{l}\text { Take } 1 \mathrm{~mL} \text { and, right after, centrifuge the } \\
\text { tubes }\left(16,000 \mathrm{~g}, 2 \text { min at } 4^{\circ} \mathrm{C}\right) \text { to remove } \\
\text { and discard the supernatant, and keep } \\
\text { the bacterial pellet. The tubes must be } \\
\text { stored at }-80^{\circ} \mathrm{C} \text { as soon as possible }\end{array}$ \\
\hline Metatranscriptomics & $\begin{array}{l}\text { Explore gut microbial gene } \\
\text { expression }\end{array}$ & $\begin{array}{l}\text { Sample fecal inoculum }{ }^{\text {a }} \text {, weigh blank } \\
\text { tube after incubation }{ }^{a} \text {, weigh sample } \\
\text { tube after incubation }{ }^{a} \text {, take samples } \\
\text { every } X \text { hours during incubation }\end{array}$ & $\begin{array}{l}\text { Obtain bacterial pellet as for } 16 \mathrm{~S} \text { rRNA } \\
\text { amplicon sequencing, and store it in } \\
\text { RNAlater to preserve RNA at }-80^{\circ} \mathrm{C}\end{array}$ \\
\hline $\begin{array}{l}\text { Bacterial enzymes activity } \\
\text { (beta-glucosidase, beta- } \\
\text { glucuronidase, } \\
\text { tryptophanase, urease) }\end{array}$ & $\begin{array}{l}\text { Check the activity of } \\
\text { potentially harmful enzymes }\end{array}$ & $\begin{array}{l}\text { Sample fecal inoculum }{ }^{\text {a }} \text {, weigh blank } \\
\text { tube after incubation }{ }^{\text {, }} \text {, weigh sample } \\
\text { tube after incubation }{ }^{a} \text {, take samples } \\
\text { every } X \text { hours during incubation }\end{array}$ & $\begin{array}{l}\text { As for } 16 \mathrm{~S} \text { amplicon sequencing or } \\
\text { shotgun metagenomic }\end{array}$ \\
\hline
\end{tabular}

${ }^{\mathrm{a} M a n d a t o r y ~ s a m p l i n g s}$

\section{Advantages and limitations}

Batch in vitro fermentations are the simplest methodology to simulate colonic fermentation. This methodology allows the assessment of as many substrate samples as the investigator chooses, either actual food, cooked or raw, or specific food components such as dietary fibers or phenolic compounds. It also allows investigators to study the behavior of healthy or altered gut microbiota against the same substrate at the same time, which could help formulate initial hypotheses and plan future experiments. Furthermore, by exposing gut microbes to certain compounds, batch fermentations can help elucidate the metabolic routes involved and the intermediate metabolites appearing. Therefore, this could in turn give clues on how to drive microbial metabolism toward a specific goal (e.g., production of a particular beneficial metabolite or favoring the growth of a beneficial bacterium), getting a step closer to gut microbiota modulation via diet. Moreover, batch fermentation experiments are much less time consuming than in vitro continuous systems or animal or human studies. In addition, whereas continuous systems usually require a big space for the vessels to simulate the different portions of the colon, batch fermentations do not. The costs of batch experiments are lower 
since they do not require certain equipment such as $\mathrm{pH}$ controlling systems or pumps to move the fermentation medium from one vessel to the next, or the additional costs from working with animal models or human volunteers. Additionally, another advantage over animal models is that animals' gut microbiota is different to that of human beings (even if gnobiotic animals are used). Therefore, because some human gut bacteria species are missing from animals' gut microbotia, and there are other species only present in animals' guts, bacterial interactions could be different, as could the effect of a specific food or food component.

However, batch in vitro fermentations also have some limitations. The main issue is that this methodology is the farthest from physiological conditions, and hence, results should not be considered definitive. $\mathrm{pH}$ is not controlled during fermentation will therefore change during the process owing to the acidic metabolites generated. As batch fermentations are not continuous systems, the accumulation of microbial waste products cannot be controlled, and bacterial growth could potentially be affected. Moreover, because of the larger number of test tubes usually used in batch fermentations, which involves more pipetting and more manipulation of the samples, microbial contamination is probably more likely in this kind of experiment.

Nonetheless, batch fermentations are still essential to study different foods or food components, and to make initial screenings that would be otherwise unfeasible because of the time required in the case of continuous systems or the costs in the case of animal and human studies. Furthermore, batch fermentations are essential to elucidate microbial metabolism of specific nutrients and, therefore, to understand the effects of incorporating certain components in the human diet.

\section{Applications}

One of the main applications of in vitro batch fermentations is to study microbial degradation of specific dietary compounds to uncover which metabolites are released and, hence, how the host health could be affected. Polyphenols have been some of the most studied compounds in this sense (Table 1). Some phytochemicals, especially phenolic compounds, are only partially absorbed in the small intestine, therefore reaching the large intestine ${ }^{24}$. Thus, in vitro batch fermentations become essential to rapidly test how these compounds are metabolized by gut microbes. Most dietary polyphenols are metabolized in the colon by gut microbes. This metabolism is usually mandatory for their absorption, and it can modify or modulate their actual biological activity ${ }^{25}$. Even though human enzymes will not break down the phytochemical structure, prior in vitro digestion is still recommended because changes in $\mathrm{pH}$ during digestion and salts could chemically alter the phytochemical structure. Additionally, the enzymes and bile salts (which can be used by gut microbes) will be present. Therefore, prior in vitro digestion will make the experiment more physiologically relevant, even though phytochemicals are not digested as carbohydrates, proteins or fats would be. Phenolic compounds have been associated with different beneficial health effects, such as antioxidant, antiinflammatory, neuroprotective and cancer chemoprotective effects ${ }^{24}$. Most polyphenols are present in foods as glycosides, while others are polymeric molecules (anthocyanins, ellagitannins) that are poorly active and must be converted into their aglycones or to monomers ${ }^{25}$. The first metabolism steps usually follow a specific pathway, and a consortium of microbes is needed for their complete degradation. However, it is also important to take into account interindividual variability, which can lead to different outcomes (different metabolites and/or physiological effects). Interindividual variation refers to the fact that different individuals can harbor different microbes, leading to variations in dietary response. Some dietary components require specific bacterial species (commonly known as keystone species) to be metabolized, and hence, if not present, these compounds would remain intact. Therefore, what is beneficial for one person could be less positive or even useless for another.

Two examples are widely studied in the field of phenolic compounds. One is metabolism of the soy isoflavone daidzein, which can be metabolized following two different pathways, depending on the gut microbes of the host ${ }^{25}$. The other example is metabolism of ellagitannins, which in most of the population leads to production of urolithin (3,8-dihydroxy-urolithin, commonly known as urolithin A and/or 3-hydroxy-urolithin, commonly known as urolithin B); in a smaller percentage of individuals, there is no urolithin production and therefore no beneficial effect from ellagitannin consumption $^{26}$. Although there is still much to unravel in relation to interindividual variability, in vitro fermentations can help identify the potential keystone species needed for the metabolism of specific dietary components, which is a first step toward predicting whether a specific person will be able to benefit (and how) from consuming a specific food component. 
Many studies have been carried out to investigate phenolic metabolism by gut microbes. Saura-Calixto et al. $^{27}$ studied microbial degradation of proanthocyanidins, discovering several phenolic acids as metabolites, which were also detected in plasma; these authors suggested that microbial degradation of proanthocyanidins would result in absorbable metabolites with potential health effects ${ }^{27}$. Ludwig et al. ${ }^{11}$ studied the catabolism of coffee chlorogenic acids by gut microbes, which allowed them to detect the pathways involved and the main metabolites derived from such phenolic compounds. These authors also demonstrated that chlorogenic acid metabolism was influenced by interindividual gut microbiota variation. Other phenolic compounds studied were flavan-3-ols ${ }^{28}$, rutin ${ }^{29}$, flavonols, flavones, flavanones and phenolic acids ${ }^{30}$, quinic acid ${ }^{31}$ and anthocyanins $^{32}$. Phenolic metabolism by gut microbes has been extensively studied; most of the current knowledge has been summarized by Selma et al. ${ }^{24}$, Serra et al. ${ }^{30}$, Marín et al. ${ }^{33}$, Stevens and Maier $^{34}$, and Rowland et al. ${ }^{25}$, among others.

Batch fermentations have also greatly facilitated the mapping of microbial metabolic pathways. This information has been successfully used to predict gut microbial metabolic outcomes after certain conditions, such a specific $\operatorname{diet}^{35}$. In addition, batch fermentations have also been used to study the effect that specific foods have on gut microbiota community structure and its functionality, which is usually measured through short-chain fatty acid (SCFA) production analysis. Their generation and relative abundance are considered health biomarkers. Individual SCFAs have been linked to several health benefits that have been already reviewed ${ }^{1,36}$. In a previous paper, we studied how green and roasted coffee could affect microbial composition and functionality, allowing us also to observe that each type of coffee affected gut microbiota differently ${ }^{18}$. In another study, we investigated the effects of chicken, chickpeas, bread, banana and pepper on gut microbiota and its functionality, observing how each type of food promoted the growth of specific bacteria ${ }^{17}$, probably due to the different composition of each food. In the same study, we observed how the cooking method applied also modified the way gut microbes metabolized such food, resulting in somewhat different microbial communities and functionalities. Other research projects have studied how meats ${ }^{37}$, legumes and insects $^{38}$ cooked differently affected gut microbiota. Moreover, not only can microbial communities and their functionality be studied, but also other biological activities such as inflammatory or antioxidant capacity after fermentation of specific foods ${ }^{16}$.

Harmful enzymatic activity of the gut microbiota is another example of an application that has been reported. This involves a set of bacterial enzymes involved in the metabolism of different substances that have as output potentially harmful metabolites ${ }^{39}$, including beta-glucosidase, beta-glucuronidase, tryptophanase, urease, azoreductase and nitroreductase. They are involved in creating aromatic amines, aglycones, secondary bile acids, hydrogen sulfide or oxygen species ${ }^{40}$.

Moreover, an important field in which batch fermentations are essential is the search for prebiotic compounds ${ }^{41}$. Accordingly, many compounds have been submitted to in vitro colonic fermentation to study their potential prebiotic effect: manooligosaccharides from spent coffee grounds ${ }^{20}$, melanoidins from different food sources ${ }^{21}$, exopolysaccharides ${ }^{2}$, maltopolysaccharides ${ }^{42}$, and inulin, galacto- and xylooligosaccharides ${ }^{43}$. All these studies would help in the screening for prebiotic ingredients, which could be added to certain foods to formulate functional foods. Once a novel prebiotic food has been formulated, it could be submitted to in vitro colonic fermentation to study its potential as a prebiotic food. In this sense, in a previous work, our research group designed a prebiotic salami in which several potential prebiotic ingredients were tested ${ }^{19}$. As result, we selected the best prebiotic ingredient, and the improved salami was tested in a human intervention ${ }^{44}$. This is a clear example of how batch fermentations are essential for initial screenings, making future interventions easier.

Finally, batch fermentations still have a critical contribution in projects focused on achieving personalized nutrition according to a person's gut microbiota. Current attempts to modulate gut microbiota via diet are usually based on genome-based metabolic reconstructions ${ }^{45}$. However, though these reconstructions are incredibly informative and useful ${ }^{46}$, they can only tell so much; carrying a gene does not mean that it is going to be expressed. They cannot account for gene expression in response to environmental changes such as $\mathrm{pH}$ or competition for a substrate ${ }^{47}$. Moreover, they usually misrepresent ecological interactions, overestimating mutually beneficial ones ${ }^{47}$. Therefore, batch fermentations become essential as an intermediate step to determine bacterial roles and gene expression in response to specific substrates. 


\section{Alternative methods}

Alternative methods to batch in vitro fermentations consist of scaling up the model to continuous systems (with or without immobilized feces), animal models and human trials. Continuous systems usually comprise three vessels mimicking the environment in the proximal, transverse and distal colon in terms of $\mathrm{pH}$, temperature and medium flow rate control ${ }^{7,9}$. The control of these parameters allows investigators to achieve a steady microbial composition as well as a steady metabolite concentration $^{48}$. Therefore, conditions are closer to those occurring in the human colon, making experiments more physiologically relevant. A widely used continuous system is $\mathrm{SHIME}^{8}$, which also includes two previous compartments to mimic gastric and intestinal digestion. However, they present some disadvantages: only one substrate (food or a specific molecule) can be tested at a time, which would make it impossible to perform initial screenings, test different foods or investigate metabolic pathways of different molecules; they require a large space to set up all the compartments and additional equipment to control the different parameters; the inoculation is usually performed through a liquid fecal suspension, which usually leads to a rapid washout of less competitive bacteria, limiting the operation time to less than 4 weeks ${ }^{48}$. To overcome these limitations, several systems have been developed. Wiese et al. ${ }^{10}$ developed a continuous system called CoMiniGut, which works with volumes as low as $5 \mathrm{~mL}$ under controlled conditions, solving the space limitations. On the other hand, there are also systems with immobilized feces where gut microbes are suspended within a porous polysaccharide matrix, overcoming the problem of using liquid fecal suspensions, with an operation time of up to $71 \mathrm{~d}^{48}$. Still, these two systems cannot handle many samples at once, and hence, batch fermentations are irreplaceable when the aim is to investigate different foods or molecules. In addition, continuous systems are much more expensive and time consuming. Therefore, batch cultures could be used for initial screenings, and continuous systems to more closely study one or a few selected compounds.

Secondly, animal models (especially gnobiotic mice) are also used to investigate gut microbiota. However, the data obtained should be interpreted with caution owing to the physiological differences between animals and humans ${ }^{49}$. Human trials/interventions are the 'gold standard', although they are expensive and are limited by social and ethical issues ${ }^{50}$. Therefore, we feel that the best approach is to combine in vitro and in vivo models ${ }^{48}$. For example, use batch culture for initial trials and screenings, then scale up to continuous systems and, finally, animal/human models.

\section{Experimental design}

\section{Prior in vitro gastrointestinal digestion}

Foods do not reach the colon in the same state that they were in when eaten. During digestion, food integrity is compromised and vegetable/animal cells are broken down, releasing their contents. Moreover, starch, proteins and fats are hydrolyzed into smaller molecules. Therefore, to mimic gut microbes' action on foods or molecules, an in vitro gastrointestinal digestion should be previously performed. We recommend the use of the protocol described by Brodkorb et al. ${ }^{15}$. Hence, we use as fermentation substrate the nondigested residue left after the intestinal digestion phase of the Brodkorb et al. INFOGEST protocol ${ }^{15}$. It has, however, been estimated that, on average, $10 \%$ of the potentially absorbable content of the small intestine is not absorbed and does reach the large intestine. Therefore, to further mimic what reaches the colon, we add $10 \%$ (vol/vol) of the intestinal soluble fraction, along with the nondigested solid residue, as fermentation substrate. If the fermentation is not going to be performed the same day as the digestion, the solid residue should be stored at $-20^{\circ} \mathrm{C}$ or below, along with aliquots of the intestinal soluble fraction enough to add $10 \%$ to all the fermentation samples. A typical fermentation example with quantities and volumes added is described in Table 2.

Stage 1: fecal material collection (Step 1)

Fecal material should be collected from human volunteers rather than from animals since, as stated before, gut microbiota varies between humans and animals ${ }^{42}$. Moreover, to overcome interindividual variability $^{5}$, it has been recommended that feces be collected and pooled from at least four volunteers ${ }^{41}$. Pooling will ensure that keystone microbes are not missing, which could result in compounds not being metabolized. One example is Ruminococcus bromii, which is needed for resistant starch degradation; if it is absent, resistant starch will not be degraded. Further examples can be found in the case of polyphenols; daidzein can be metabolized following two different pathways depending on the gut microbes of the host ${ }^{25}$, and ellagitannins that are metabolized by some microbes that are not present in a small percentage of the general population ${ }^{26}$. However, it is important to note that if the 
aim of the experiment is to study a specific microbial community (i.e., an individual's microbial community), then the pooling strategy would not be ideal since the objective would be, for instance, to determine whether that individual is able to metabolize ellagitannins. To use the protocol to test the effect of an individual food on different fecal samples (i.e., feces from different donors, assessed individually rather than being pooled), feces would need to be weighed in individual tubes as soon as they are received in the laboratory and frozen, before being defrosted on the day of the fermentation and mixed with the digested food to be tested. Although we plan to do this in future, we have not yet tested this approach, and it is therefore outside the scope of this protocol.

When the aim is to investigate how gut microbes from healthy people are affected by specific foodstuffs or molecules, feces should be collected from healthy volunteers who are not overweight (body mass index within normal range $)^{51}$, have had no antibiotic treatment in the last 6 months ${ }^{52}$, have no intestinal conditions ${ }^{53}$, belong to the same age range, follow similar diets and are exposed to similar environments ${ }^{54}$. Batch fermentations could be also used to study gut microbiota from people with conditions such as colorectal cancer, inflammatory diseases, obesity or celiac disease. Fecal material must be always collected under sterile conditions - using a sterile container, gloves and a disposable sterile spoon (another option is the use of stool collectors, e.g., the Fecotainer, AT Medical). Therefore, proper instructions should be provided to the volunteers. Fecal material collection should be planned in advance to make sure that feces are available on the desired day to perform the experiment.

When possible, fecal samples should be collected and used for inoculum preparation within $1 \mathrm{~h}$ after collection, keeping them at $4{ }^{\circ} \mathrm{C}$ or on ice ${ }^{55}$. This would avoid substantial changes in the metabolic profile ${ }^{55}$ and bacterial taxa abundance ${ }^{56}$. However, this is not always possible. Keeping the stool samples at $4{ }^{\circ} \mathrm{C}$ or room temperature for longer periods of time would affect metabolic and bacterial abundance profiles ${ }^{56}$. And, although freezing the fecal material will compromise cell viability $^{55}$, adding glycerol as cryoprotectant can help preserve bacteria. Therefore, since usually all fecal material needed will not be collected within $1 \mathrm{~h}$ before the experiment, we think the best option is to freeze it as standard procedure. This could happen, for example, when fecal material from at least four volunteers is needed and one of them was not able to provide it because of physiological (or other) reasons. Still, the fecal material collected should be from that morning. Once received in the laboratory, it must be mixed with $20 \%$ (wt/vol) glycerol in proportion 50:50 and frozen at $-80{ }^{\circ} \mathrm{C}$. The samples can be transported to the laboratory in a cooler bag if the distance is short (e.g., in the same city), always ensuring that the container is properly closed. If the distance is larger, they should be transported on dry ice.

Stage 2: equipment and reagent preparation (Steps 2 and 3)

In this stage, all the equipment and reagents to be used are autoclaved: pipette tips $(1 \mathrm{~mL}, 10 \mathrm{~mL})$, laboratory spoons, and tubes. It is also important to autoclave Milli-Q water to make up for the volume of medium and buffer lost to evaporation during autoclaving.

In relation to medium preparation, there are two main options: phosphate-buffered saline (oligotrophic) and basal culture medium (eutrophic) (Table 1). Oligotrophic fermentations are inoculated with a higher concentration of fecal inoculum (5-30\%, wt/vol) as a source of nutrients and microbes, whereas eutrophic fermentations are inoculated with a lower fecal inoculum concentration $\left(\sim 1 \%\right.$, wt/vol) into a basal culture ${ }^{54,57}$. Typical eutrophic medium is composed of peptone water, yeast extract, bile salts, cysteine, vitamin $\mathrm{K}$, hemin and several salts, whereas typical oligotrophic medium is only composed of several salts and cysteine ${ }^{57}$. Some authors, however, add peptone water in addition to the salt mix in oligotrophic mediums (Table 1), as a source of additional nitrogen. Nevertheless, it has been suggested that additional nitrogen is not usually needed since it is provided in sufficient quantity by the fermentation substrate and the inoculum ${ }^{14}$. According to Long et al. ${ }^{57}$, the microbial communities resulting from eutrophic mediums are similar to those observed in animal and human studies receiving high-fat/high-protein diets. These microbial communities are characterized by high abundance of Escherichia/Shigella and low abundance of Faecalibacterium (as opposed to those diets characterized by high plant consumption), an increase of bile-tolerant bacteria and a decrease of SCFA-producing genera. On the other hand, the oligotrophic medium leads to a community similar to that observed in feces of humans on a normal or calorie-restricted diet. In this protocol, we propose the use of a $32 \%$ (wt/vol) inoculum, as it has been used in our previous works with success, as well as by other authors ${ }^{11,17-21,28,29}$ along with an oligotrophic medium with peptone.

This stage should be carried out the day before performing the experiment, so all equipment is ready and buffers and media have cooled down enough to be used. Cysteine is sensitive to 
temperature, so it must be added to the medium after the medium has been autoclaved. As cysteine cannot be autoclaved but still needs to be sterile, a filtration step could be performed after its addition.

Stage 3: setting up samples from in vitro digestion for in vitro fermentation (Steps 4 and 5)

First, it is important to run a blank in parallel to control for the effect of the fermentation medium on gut microbial behavior. This tube will have an equivalent volume of Milli-Q water added instead of the actual sample (i.e., food or a food component). This control tube will be called control tube A. Additionally, another control tube for in vitro fermentation will be needed (control tube B). This tube will carry the same components as control tube A and sample tubes, but instead of the actual sample or Milli-Q water (as in tube A), it will carry the solid residue from the control tube coming from in vitro digestion. This is important since the latter will contain the salts and enzymes used for the digestion, which could be used by gut microbes (see 'Control tubes').

The solid residue (undigested fraction) from the previous in vitro digestion will be used as sample. To collect this solid residue, in vitro digestion tubes must be centrifuged at $4,000 \mathrm{~g}$ for $10 \mathrm{~min}$ at $4{ }^{\circ} \mathrm{C}$. The supernatant can be stored for analysis or discarded depending on the needs of the experiment, as described previously ${ }^{15}$. We recommend fermenting $0.5 \mathrm{~g}$ of solid residue to ensure that there is enough sample for bacteria to ferment ${ }^{16}$. Additionally, because it is known that (on average) $10 \%$ of the supposedly absorbable fraction in the large intestine is actually not absorbed, we add the corresponding volume of the in vitro digestion supernatant (absorbable fraction reaching the large intestine). This volume is calculated as follows: we first calculate $10 \%$ (vol/vol) of the supernatant, which corresponds to the total amount of solid residue (undigested fraction) available from that digestion reaction. As we will only ferment $0.5 \mathrm{~g}$ of solid residue, we then calculate the proportional volume of supernatant to add. Example: if the in vitro digested reaction has $3 \mathrm{~g}$ of solid residue and $37 \mathrm{~mL}$ of supernatant, $10 \%$ of supernatant is $3.7 \mathrm{~mL}$, which would correspond to $3 \mathrm{~g}$ of solid residue. So, to ferment $0.5 \mathrm{~g}$ of solid residue, we would add $0.5 \mathrm{~g} \times 3.7 \mathrm{~mL} / 3 \mathrm{~g}=0.62 \mathrm{~mL}$ of supernatant.

\section{Stage 4: fecal slurry preparation (Step 6)}

Here, we use a $32 \%$ (wt/vol) inoculum (fecal slurry) in phosphate buffer adjusted to $\mathrm{pH} 7.0$ with $\mathrm{HCl}$ $0.1 \mathrm{M}$ (instructions for preparation in 'Reagent setup'). With 32\% (wt/vol) of feces, we ensure an appropriate bacterial cell density, and the inoculum can function as both a source of microbes and a source of nutrients for the microbes, instead of using a more complex medium. To prepare the inoculum, if the feces were frozen, they must first be thawed at room temperature. Feces manipulation must be performed in an anaerobic chamber $\left(80 \% \mathrm{~N}_{2}, 10 \% \mathrm{CO}_{2}\right.$ and $\left.10 \% \mathrm{H}_{2}\right)$. Once thawed, glycerol must be removed by centrifuging at $4,000 \mathrm{~g}$ for $10 \mathrm{~min}$ at $4{ }^{\circ} \mathrm{C}$, keeping the pellet and discarding the supernatant. The fecal pellet is resuspended in phosphate buffer $0.1 \mathrm{M} \mathrm{pH} 7.0$ at a concentration of $32 \%$ fecal pellet:phosphate buffer (wt/vol). Immediately afterward, once the feces have been mixed with the phosphate buffer, they have to be properly homogenized using a vortex for $1 \mathrm{~min}$. Secondly, the fecal suspension should be centrifuged to remove larger particles (550 $g$ for $5 \mathrm{~min}$ at room temperature). Since this centrifugation is only to remove large particles, we now keep the supernatant, which contains bacterial cells. This supernatant is the fecal inoculum. The fecal inoculum is then added to each fermentation tube at $20 \%(\mathrm{vol} / \mathrm{vol})$ in relation to the fermentation medium and substrate sample to be tested (Step 7). This concentration was tested previously ${ }^{16}$, observing how with $20 \%$ (wt/vol) of inoculum a higher degradation of the substrate was achieved than with $10 \%(\mathrm{wt} / \mathrm{vol})$, which resulted in a higher antioxidant capacity release. We recommend preparing 1.5 or $2 \times$ the inoculum volume needed. For instance, for ten fermentation tubes, the inoculum volume needed would be $20 \mathrm{~mL}$ ( $2 \mathrm{~mL}$ each, if final tube volume is $10 \mathrm{~mL}, 20 \% \mathrm{vol} / \mathrm{vol}$ ). However, the inoculum volume to be prepared should be $30-40 \mathrm{~mL}$, as after centrifuging the fecal suspension, some volume will remain in the sedimented pellet and therefore will not be accessible; thus, some extra volume should be prepared.

During this stage, it is important to take samples of the fecal slurry to determine baseline conditions, which will be needed for subsequent data analysis. Baseline sampling should be performed according to the aim of the experiment and subsequent analysis methods that will be used (e.g., metabolomics, 16S rRNA or amplicon sequencing, shotgun metagenomics, metatranscriptomics). Examples of further analysis usually performed and initial baseline sampling strategies are listed below.

- Metabolomics analysis: the aim will be to measure the presence of certain metabolites. Typical metabolites measured as result of in vitro fermentation are SCFAs. In this case, it is enough to take 1 $\mathrm{mL}$ of the fecal slurry into a tube and store it at $-80{ }^{\circ} \mathrm{C}$ (for no longer than a month because some molecules, such as some polyphenols, can break down after prolonged storage). 
- 16S rRNA amplicon sequencing or shotgun metagenomics analysis: here it is especially important to keep sterile conditions to avoid contamination with environmental bacteria, or bacteria from the researcher. It is enough to take $1 \mathrm{~mL}$ of the fecal slurry. Right after, the tubes must be centrifuged $(16,000 \mathrm{~g}, 2 \mathrm{~min})$ to remove the supernatant and keep the bacterial pellet. The tubes have to be stored at $-80{ }^{\circ} \mathrm{C}$ as soon as possible. Samples stored at $-80{ }^{\circ} \mathrm{C}$ are stable for $3-6$ months.

- Metatranscriptomics analysis: the bacterial pellet must be stored in RNAlater for RNA preservation and kept at $-80{ }^{\circ} \mathrm{C}$ for up to 1 month.

- Measurement of activity of potentially harmful microbial enzymes (beta-glucosidase, betaglucuronidase, tryptophanase, urease): obtain the bacterial pellet as for $16 \mathrm{~S}$ rRNA amplicon sequencing or shotgun metagenomics and store it at $-80{ }^{\circ} \mathrm{C}$ for up to 3 months.

Stage 5: fermentation (Steps 7-9)

The fermentation involves mixing the following different components into sterile tubes: medium, inoculum (fecal slurry), sample from in vitro digestion (substrate), and $10 \%$ (vol/vol) of the digestion supernatant volume. This process should be performed under anaerobic conditions in an anaerobic workstation $\left(80 \% \mathrm{~N}_{2}, 10 \% \mathrm{CO}_{2}\right.$ and $\left.10 \% \mathrm{H}_{2}\right)$. The amount of substrate and volume of fecal slurry were previously tested ${ }^{16}$. Once the different components have been added to the tube, $\mathrm{N}_{2}$ is bubbled for $1 \mathrm{~min}$ and tubes are placed at $37{ }^{\circ} \mathrm{C}$ with oscillation at $20 \mathrm{rpm}$ for $20 \mathrm{~h}$. The final volume inside the fermentation tube is $10 \mathrm{~mL}$ plus the $10 \%$ of the volume of the in vitro digestion supernatant, so a $15 \mathrm{~mL}$ tube would be sufficient. However, owing to gas production during fermentation, a bigger headspace is needed and, hence, $50 \mathrm{~mL}$ tubes are recommended.

\section{Stage 6: sampling and storage (Step 10)}

Once incubation has finished, to stop microbial fermentation the tubes are submerged in ice for $15 \mathrm{~min}$, and aliquots are taken as needed at the end of the $15 \mathrm{~min}$, keeping the tubes in the ice (Table 3). Additionally, samples can be taken during fermentation at desired timepoints if, for instance, the researcher wants to study the production of a certain metabolite over time. In this case, tubes must not be submerged in ice, and anaerobic as well as aseptic conditions must be kept while sampling. Sampling should be performed according to the aim of the experiment and subsequent analysis (metabolomics, 16S rRNA amplicon sequencing or shotgun metagenomics, metatranscriptomics, etc.), as described above for baseline sampling (see 'Experimental design', Stage 4). Further information about the sampling procedure can be found in Table 3.

\section{Stage 7: sample processing (Step 11)}

Since the water content of the sample will change during the prior in vitro digestion (digestion will usually increase water content of the sample, resulting in a solid residue with a lower percentage of solid matter than the original food), a critical point is to measure the water content of the original food sample (if it is food), the undigested solid residue and the unfermented solid residue (if any). By calculating the water content, we will know the amount of solid matter submitted to digestion and fermentation and what is left after fermenting, which will allow us to go back in the calculations and determine the amount of actual digested and fermented food. Water content measurements are useful to express results (for instance, metabolite concentration) per unit of mass of the original food and, secondly, to know the amount of digested food used by gut bacteria. Water content of original food or undigested solid residue can be measured by weighing $1 \mathrm{~g}$ of each and heating to $100{ }^{\circ} \mathrm{C}$ on a stove for $2 \mathrm{~h}$, or longer (samples with high water content could need up to $3 \mathrm{~h}$ ). Water content can be calculated by determining the weight loss (see Step 11A). However, to measure the water content of the unfermented residue, we recommend lyophilization, which is more expensive since this equipment is not available in all laboratories, but may be necessary as there will be very little residue after sampling.

Sample processing will depend on the analysis to be performed afterward. As described in 'Anticipated results', potential purposes of in vitro fermentation include the following:

- Investigation of substrate degradation. These data will elucidate how much of a given food has been degraded by the gut microbial populations. See Step 11A for details

- Investigation of the metabolism of phytochemicals and gut microbial functionality. Here we will rely on metabolomics analysis. Some typical metabolites measured as result of in vitro fermentation are SCFAs and polyphenols. Microbial metabolization of phenolic compounds has been extensively studied. Since most phenolic compounds are not absorbed in the small intestine and reach the colon, 
the data obtained using in vitro fermentation will enable study of how they are metabolized by the gut microbiota and which metabolites are available for absorption in the large intestine. SCFAs are the main metabolites resulting from microbial fermentation of food, especially fiber, and have proven to be beneficial for human health, and their production from given foods is important to understand for dietary advice purposes. Prior to metabolomics analysis, sampled tubes are centrifuged and the supernatant is treated as described in Step 11B

- Investigation of gut microbial community structure. Depending on the technology used here-16S rRNA amplicon sequencing or shotgun metagenomics analysis (enabling analysis of the whole bacterial genome)-the dataset will provide phylogenetic information up to genus or species, respectively. The first scenario is the most commonly used since it is cheaper and these experiments are usually initial screenings, with the aim to select specific conditions for further exploration. However, 16S RNA data also have disadvantages, as has been reported when screening for prebiotics ${ }^{57}$. Sometimes prebiotics have an effect only on certain species of the same genus, which 16S RNA is not usually able to detect because the species that do not change mask the affected ones. There are, however, bioinformatic approaches that can estimate up to species level. Regardless of the approach, such investigations generate a huge amount of data, which makes their analysis difficult. Therefore, a proper methodology to obtain meaningful and valid conclusions must be utilized. Paliy and Shankar ${ }^{58}$ reviewed the main statistical approaches available to interpret genomic data

Data obtained in such investigations will provide information about the different bacteria present in the gut. Many of them have been linked to either beneficial or detrimental effects on human health. Thus, investigating which microbes can be favored or inhibited by given foods could prove essential for dietary advice. For $16 \mathrm{~S}$ rRNA amplicon sequencing or shotgun metagenomics analysis, sample preparation is described in Step 11C.

\section{Control tubes}

Control tubes are used to control for the effect of anything that is not the sample (i.e., the food or food component) being studied. The following control tubes should be prepared:

- Control tube from in vitro digestion. Its preparation is thoroughly described in the in vitro digestion protocol $^{15}$. This control tube is important because it contains all salts and enzymes, which can be used by gut microbes

- Control tube for in vitro fermentation A. This tube should carry Milli-Q water instead of the substrate sample (i.e., food or food component), in an equivalent volume. Therefore, it should contain $7.5 \mathrm{~mL}$ of fermentation medium, $2 \mathrm{~mL}$ of $32 \%$ inoculum (fecal slurry) and $0.5 \mathrm{~mL}$ of Milli-Q water. This tube will allow users to control for the effect of the fermentation medium on the gut microbiota

- Control tube for in vitro fermentation B. This tube will carry the solid residue from control tube from in vitro digestion $(0.5 \mathrm{~g}$ ) and $7.5 \mathrm{~mL}$ of fermentation medium and $2 \mathrm{~mL}$ of $32 \%$ inoculum (fecal slurry). This tube will allow users to control for the effect of the enzymes and salts used in the in vitro digestion on the gut microbiota

\section{Throughput}

One of the main advantages of this protocol is the ability to test many different foodstuffs or food components within the same experiment. Therefore, is a perfect screening tool to search for relations between bacteria and food. Examples of the numbers of food samples we tested in some of our studies are as follows: in ref. ${ }^{59}$ we tested 127 vegetable samples $\times 3$ (triplicates), performed during $7 \mathrm{~d}(\sim 50$ fermentations per day); in ref. ${ }^{17}$ we tested 15 foodstuff samples $\times 3$ (triplicates); in ref. ${ }^{18}$ coffee samples $\times 3$ (triplicates); in ref. ${ }^{19}$ salami samples $\times 3$ (triplicates); in ref. ${ }^{20}$ coffee samples $\times 3$ (triplicates); and in ref. ${ }^{21}$ melanoidin samples $\times 3$ (triplicates).

\section{Reproducibility}

Reproducibility was tested by repeating this protocol on six consecutive days to ferment lentils using fecal material from four celiac, four obese and four lean adults. The fecal material from the four individuals with each condition was pooled together to obtain three fecal slurry samples, one from each group. The in vitro fermentation protocol was run for each fecal slurry sample (i.e., three fermentations) and repeated six times on consecutive days. Each day, aliquots were taken and the gut microbial community was investigated via $16 \mathrm{~S}$ rRNA sequencing. We obtained the relative abundance (\% of each bacteria with respect to the total community) of each gut bacterium, at the genus level, present in the microbial community (i.e., we learned which bacteria were present and in which 


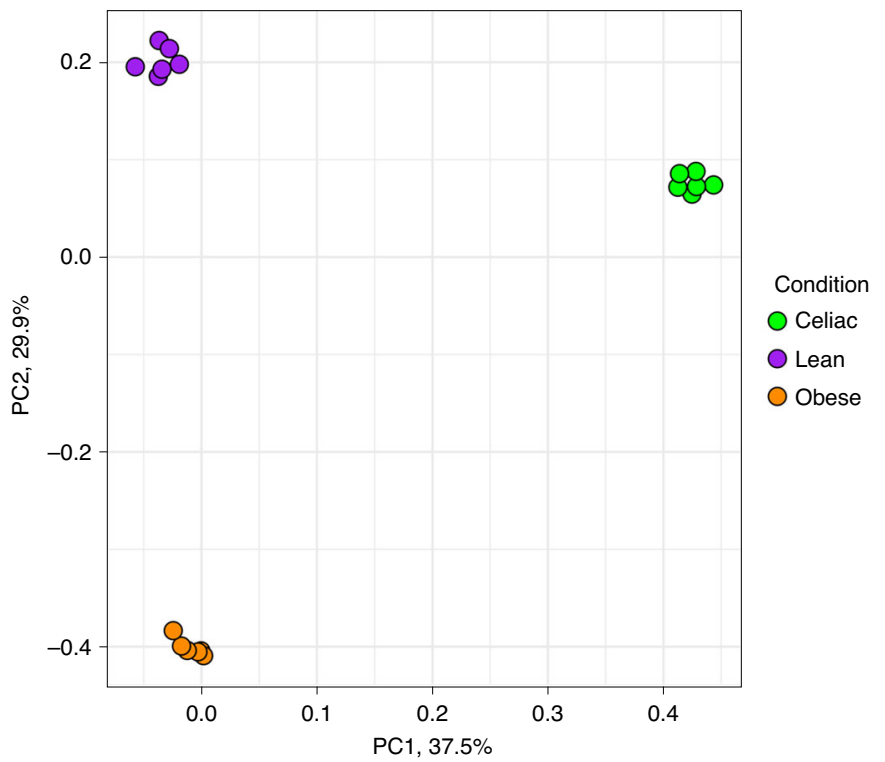

Fig. 2 | Reproducibility assessment. Principal coordinates analysis with Bray-Curtis dissimilarity distance of microbial genus abundance obtained after repeating the protocol on six consecutive days to in vitro ferment lentils using fecal material from four lean, four celiac and four obese adults. Each dot represents the gut microbial community obtained that day from lentil fermentation. Each group clustering together indicates that microbial communities were very similar across days.

proportions). To assess whether these gut microbial communities differed significantly $(P<0.05)$ from day to day, dissimilarity between samples was calculated according to the Bray-Curtis method, and results were depicted graphically on principal coordinates analysis. This approach allowed us to calculate similarity or dissimilarity between samples (i.e., from lean, obese and celiac subjects) according to a set of variables (i.e., each bacterial genus forming the microbial population). Basically, this depicts how different or similar microbial communities from each day are. As depicted in Fig. 2, samples are depicted as dots, with dots very close together belonging to the same group. This indicates that microbial communities are very similar regardless of the day the aliquot was taken, and no significant $(P<0.05)$ differences between days were found. On the other hand, the large distance observed between groups demonstrates how different microbial communities from lean, obese and celiac people can be. This protocol is able to reflect such differences.

Materials

\section{Biological materials}

- Fecal material ! CAUTION Use proper personal protective equipment and work in an anaerobic chamber while handling feces. When possible, use sterile plastic material that can be discarded afterward. These materials used for feces handling and inoculum preparation must be discarded in a biological hazards container. Material not to be discarded should be washed with bleach (10-20\%) and/or autoclaved. ! CAUTION Ethical approval to work with human feces must be obtained from the Ethics Committee of the interested institution. A fecal sample volunteer information sheet and collection consent must be prepared.

\section{Reagents}

- Ultrapure type I water, generated by a Milli-Q system or similar

- Sodium phosphate monobasic dihydrate (Merck, cat. no. 567550)

- $\mathrm{NaOH}$ (Merck, cat. no. 9141) ! CAUTION $\mathrm{NaOH}$ is corrosive and causes severe skin burns and eye damage. Use proper personal protective equipment.

- $\mathrm{HCl}$ (J.T. Baker, cat. no. 6081) ! CAUTION HCl is corrosive, causes burns and is irritating to the respiratory system. Use proper personal protective equipment and work in a fume hood while handling it.

- Peptone (Sigma-Aldrich, cat. no. T7293) 
- L-Cysteine (Sigma-Aldrich, cat. no. 168149)

- Resazurin sodium salt (Sigma-Aldrich, cat. no. 199303)

- Sodium sulfide hydrate (Sigma-Aldrich, cat. no. 14738)

\section{Equipment}

- Standard laboratory centrifuge suitable for $50 \mathrm{~mL}$ tubes, Sigma 2-16KL (Sigma, cat. no. 5710350)

- Standard laboratory centrifuge suitable for Eppendorf tubes, Labnet Spectrafuge 24D (Labnet, cat. no. LA-C2400)

- Standard laboratory pH meter, Laqua-PH1100 (Horiba Scientific, cat. no. 3200674407)

- Standard laboratory vortex, Select Vortexer (Select Bioproducts, cat. no. SBS100-2)

- Milli-Q water system, Synergy UV (Merck, cat. no. F2PA71772C)

- Fecal collection kits, Fisherbrand Commode Specimen Collection System (Thermo Fisher Scientific, cat. no. 02-544-208)

- Gas installation (N, carbon dioxide and hydrogen)

- Anaerobic chamber, Whitley A25 Workstation (Don Whitley Scientific, cat. no. A09031)

- Oscillator able to hold $50 \mathrm{~mL}$ tubes, IKA Rocker 2D digital (IKA, cat. no. 0004003000)

- Incubator large enough for the oscillator to fit inside, Universal Incubator Memmert UF75 (Memmert, cat. no. B318.650)

- $-80{ }^{\circ} \mathrm{C}$ freezer, Lab Care Plus (Infrico Medicare, cat. no. ULF50086)

- Basic benchtop laboratory freeze dryer, LyoQuest $-85^{\circ} \mathrm{C}$ (Telstar, cat. no. 58201)

- Eppendorf tubes, $1.5 \mathrm{~mL}$ (Deltalab, cat. no. 200400P) and $2 \mathrm{~mL}$ (Deltalab, cat. no. 4092.6N)

- Centrifuge tubes, $50 \mathrm{~mL}$ (Deltalab, cat. no. 429926)

- Micropipettes, Gilson P1000 (Gilson, cat. no. F167550) and sterile tips (Neptune, cat. no. BT1000.95)

- Glass beakers and volumetric flasks (Fisher Scientific, cat. no. 05-404-120)

- Precision balance, Radwag PS4500.X2 (Radwag Balances and Scales, cat. no. WL-212-0134)

- Analytical balance, Radwag AS 82/220.R2 (Radwag Balances and Scales, cat. no. WL-104-1051)

- Magnetic stirrer, SinerLab MS-H-Pro+ (SinerLab, cat. no. SN74915)

\section{Reagent setup}

- Phosphate buffer solution: prepare the phosphate buffer at $0.1 \mathrm{M}$ concentration, and adjust the $\mathrm{pH}$ to 7.0 with $1 \mathrm{M} \mathrm{HCl}$. Dissolve the reagent in less volume of Milli-Q water than the final volume, adjust the $\mathrm{pH}$ and then make up to the final volume with water. Phosphate buffer can be stored for up to 1 month at room temperature (always checking for salt precipitation) though $\mathrm{pH}$ has to be checked prior to use, corrected if needed, and the solution autoclaved. Sterile Milli-Q water to make up for the volume loss during autoclaving must be added

- Peptone solution: prepare the peptone solution by dissolving $15 \mathrm{~g}$ of peptone in almost $1 \mathrm{~L}$ of Milli-Q water, adjust the $\mathrm{pH}$ to 7.0 and then make up to $1 \mathrm{~L}$ with water. Make fresh before use. Autoclave before use. Volume lost during autoclaving will have to be compensated with sterile Milli-Q water. $\triangle$ CRITICAL If the volume is not corrected, the concentration of nutrients will vary and experiments will not be reproducible. In addition, it is critical to avoid contamination afterward.

- Reductive solution: prepare the reductive solution by dissolving $312 \mathrm{mg}$ of cysteine and $312 \mathrm{mg}$ of sodium sulfide in $2 \mathrm{~mL}$ of $1 \mathrm{M} \mathrm{NaOH}$, and make up the volume to $50 \mathrm{~mL}$ with Milli-Q water. Make fresh before use. $\triangle$ CRITICAL STEP Cysteine is sensitive to thermal treatment, so it cannot be autoclaved. Instead, reductive solution must be prepared in sterile conditions and under anaerobic environment $\left(80 \% \mathrm{~N}_{2}, 10 \% \mathrm{CO}_{2}\right.$ and $\left.10 \% \mathrm{H}_{2}\right)$.

- Resazurin solution: prepare resazurin solution at $0.1 \%(\mathrm{wt} / \mathrm{vol})$. To prepare this solution, weigh $1 \mathrm{mg}$ of resazurin and dissolve it in $1 \mathrm{~mL}$ of Milli-Q water. Only $1.25 \mathrm{~mL}$ of resazurin solution is needed for each liter of fermentation medium, so usually $2 \mathrm{~mL}$ of resazurin is enough. Make fresh before use. Autoclave before use. Volume lost during autoclaving will have to be compensated with sterile MilliQ water. $\triangle$ CRITICAL If the volume is not corrected, the concentration of nutrients will vary and experiments will not be reproducible. In addition, it is critical to avoid contamination afterward.

- Final fermentation medium: mix $1 \mathrm{~L}$ of peptone solution with $50 \mathrm{~mL}$ of reductive solution and $1.25 \mathrm{~mL}$ of resazurin for each liter of fermentation medium. $\triangle$ CRITICAL STEP This must be carried out under anaerobic and sterile conditions to avoid contamination. 
In vitro fermentation Timing 5-7 $\mathrm{h}$ to prepare the equipment, reagents and tubes before incubating, plus $20 \mathrm{~h}$ of incubation and $1 \mathrm{~h}$ for sampling (estimated for 50 fermentation tubes)

1 Fecal material collection. Fecal material must be always collected using sterile conditions: sterile container, using gloves and with help of a disposable sterile spoon. Another option is the use of stool collectors (e.g., the Fecotainer, AT Medical). Once collected, fecal material can be stored by the volunteer in their home refrigerator and transported to the laboratory in a cooler bag within $24 \mathrm{~h}$. Upon arriving at the laboratory, mix the feces with a water:glycerol solution $(20 \% \mathrm{vol} / \mathrm{vol})$ and store at $-80^{\circ} \mathrm{C}$. For detailed instructions see 'Experimental Design', Stage 1.

2 Reagent setup. Prepare reagents, including phosphate buffer $0.1 \mathrm{M} \mathrm{pH} 7$ for fecal slurry preparation and fermentation medium. Reagents should be prepared the day before the experiment as well as autoclaved. For detailed instructions on preparing the different reagents, see 'Experimental design', Stage 2, and 'Reagent setup'.

$\triangle$ CRITICAL STEP Note that cysteine cannot be autoclaved as it is heat sensitive.

3 Equipment setup. Autoclave all utensils that will be used. This should be performed the day before the experiment, keeping utensils wrapped in foil after autoclaving. For detailed instructions, see 'Experimental design', Stage 2, and 'Equipment'.

4 Fermentation setup. Weigh the substrate samples into their corresponding $50 \mathrm{~mL}$ tubes. The amount of sample to be used is $0.5 \mathrm{~g}$ of the solid residue left after centrifuging the in vitro digestion reaction. Homogenization of the solid residue is needed, which can be done with a stainless steel sterile spatula

$\triangle$ CRITICAL STEP It is essential to first weigh the empty tube to calculate the remaining solid residue after fermentation.

\section{? TROUBLESHOOTING}

5 Add $10 \%$ of the in vitro digestion supernatant (see 'Experimental design', Stage 3, for instructions for calculating the amount).

6 Using the fecal sample from Step 1, prepare the inoculum at 32\% (wt/vol) feces/phosphate buffer $0.1 \mathrm{M}$ at $\mathrm{pH}$ 7.0. Right after centrifuging, we recommend that the inoculum be moved with care to another vessel so that large particles remain in the centrifuged tube. Take baseline samples from the fecal inoculum. See 'Experimental design', Stage 4, for further information.

$\triangle$ CRITICAL STEP It is especially important to keep conditions as sterile and anaerobic as possible to avoid contamination and death of strictly anaerobic bacteria.

$\triangle$ CRITICAL STEP Do not centrifuge above 550g in order to avoid sedimentation of bacterial cells.

7 Under anaerobic $\left(80 \% \mathrm{~N}_{2}, 10 \% \mathrm{CO}_{2}\right.$ and $\left.10 \% \mathrm{H}_{2}\right)$ and aseptic conditions, add $7.5 \mathrm{~mL}$ of the fermentation medium (from Step 2) and $2 \mathrm{~mL}$ of fecal inoculum (from Step 6) to each fermentation tube containing the weighed sample and supernatant (from Step 5). Bubble nitrogen for 1 min to remove any oxygen that might have entered the tube. Also set up control tubes as described in 'Experimental design'.

\section{? TROUBLESHOOTING}

8 Note the final volume added to the tube.

$\triangle$ CRITICAL STEP The information is needed for future calculations.

9 Fermentation. Incubate the tubes for $20 \mathrm{~h}$ at $37^{\circ} \mathrm{C}$ under oscillation at $20 \mathrm{rpm}$. ? TROUBLESHOOTING

10 Sampling. To stop fermentation, submerge the tubes in ice for $15 \mathrm{~min}$, then quickly agitate the tube and take aliquots, keeping the tubes in ice. Additionally, samples can be taken during fermentation at desired timepoints if, for instance, the researcher wants to study the production of a certain metabolite over time. In this case, tubes must not be submerged in ice, and anaerobic as well as aseptic conditions must be kept while sampling. Sampling should be performed, depending on the analysis, as described in Table 3 (and see 'Experimental design', Stage 6, for further details). We recommend taking $2 \mathrm{~mL}$ for each desired further experiment and storing in two separate $1 \mathrm{~mL}$ tubes at $-80{ }^{\circ} \mathrm{C}$ until analysis, eliminating the need to open and defrost the same sample more than once.

$\triangle$ CRITICAL STEP Note that such volumes are valid for the types of analyses described in this protocol, but other analyses may require larger volumes.

$\triangle$ CRITICAL STEP For sampling, it is essential to first agitate the tube to ensure that bacterial communities or compounds of interest have not sedimented during their time in ice. 
11 Sampling processing. Sampling processing should be performed, depending on the analysis, as follows. Some examples of results previously obtained using this protocol are described in 'Anticipated results'. These were obtained according to the following procedures-use option A to assess substrate degradation (Table 5), option B for metabolite analysis, or option C for shotgun metagenomics and 16S rRNA analysis (see 'Experimental design', Stage 7, for further details):

(A) Substrate degradation. (for 50 samples, $4 \mathrm{~h}$ if using a stove, $45 \mathrm{~h}$ if using lyophilization).

(i) Perform dry matter calculation on samples of the original substrate (food) submitted to in vitro digestion, the solid residue from in vitro digestion (used as the fermentation input sample) and the unfermented residue left after in vitro fermentation. To calculate the water content of samples, first weigh $1 \mathrm{~g}$ (this is not a fixed amount) of the given food tested, the solid residue obtained after in vitro digestion (fermentation input) and the solid residue left after the in vitro fermentation (unfermented residue).

(ii) Remove water content by heating on a stove at $100{ }^{\circ} \mathrm{C}$ for $2 \mathrm{~h}$ or by lyophilization. If the latter option is chosen, samples must be first frozen at $-80^{\circ} \mathrm{C}$. Once frozen, they can be introduced into the lyophilizer (quickly to prevent samples from thawing) and lyophilized following the instructions of the manufacturer. Lyophilization of $1 \mathrm{~g}$ of sample will take $\sim 6 \mathrm{~h}$ (see 'Experimental design', Stage 7, for further information).

(iii) Calculate the water content of each sample as follows: weight before drying - weight after drying $=$ water content.

(iv) Calculate the dry matter of each sample as follows: $g$ of sample - ( $g$ of sample $\times \%$ water content/100).

(v) Use the dry matter to calculate how much food has been degraded, as follows:

a Food degraded during in vitro digestion (\%): (dry matter of food submitted to in vitro digestion $(\mathrm{g})$ - dry matter of solid residue after in vitro digestion $(\mathrm{g})) \times 100 /$ dry matter of food submitted to in vitro digestion (g). See Table 5, Digested \% column.

b Food degraded during in vitro fermentation (\%): (dry matter of sample submitted to in vitro fermentation $(\mathrm{g})$ - dry matter of solid residue after in vitro fermentation $(\mathrm{g})) \times$ 100/dry matter of sample submitted to in vitro digestion (g). See Table 5, 'Fermented \%'.

c Food not degraded (final solid residue after in vitro fermentation, (\%): (dry matter of solid residue after in vitro fermentation $(\mathrm{g})) \times 100$ )/dry matter of food submitted to in vitro digestion (g). See Table 5, 'Final solid residue \%'.

(vi) Using these data, express results per unit of food digested or fermented.

(B) Metabolite analysis (for 50 samples, phenolic analysis takes $\mathbf{2} \mathbf{d}$ for extraction and $\mathbf{2} \mathbf{d}$ for HPLC analysis; for SCFA, $1 \mathrm{~d}$ to prepare the samples and $1 \mathrm{~d}$ for HPLC analysis)

$\triangle$ CRITICAL Sample processing can be very different depending on the metabolites under study. In the examples shown in 'Anticipated results', we specifically describe phenolic compounds and SCFAs.

(i) Phenolic extraction. For phenolic compounds, first extract with an organic solvent, one commonly used is diethyl ether. Although the process is described in refs. ${ }^{18,21}$, the extraction procedure is as follows: first, mix $1 \mathrm{~mL}$ of fermentation liquid with $1 \mathrm{~mL}$ of diethyl ether (relation 50:50, vol/vol) and store at $4{ }^{\circ} \mathrm{C}$ for $24 \mathrm{~h}$ in darkness.

(ii) Recover the organic fraction, and put into another clean $10 \mathrm{~mL}$ tube.

$\triangle$ CRITICAL STEP The organic fraction will be the one beneath the aqueous phase.

(iii) Again add $1 \mathrm{~mL}$ of diethyl ether to the fermentation liquid (second extraction), mix carefully and manually to avoid foam, and recover the organic fraction into the same 10 $\mathrm{mL}$ tube.

(iv) Repeat one more time, to obtain $3 \mathrm{~mL}$ of organic solvent with dissolved polyphenols.

(v) Evaporate the solvent (diethyl ether) in a rotatory evaporator with water bath $\left(\right.$ at $30{ }^{\circ} \mathrm{C}$ ), and resuspend the polyphenols in $1 \mathrm{~mL}$ of Milli-Q water:methanol 50:50 ( $\mathrm{vol} / \mathrm{vol})$.

(vi) Phenolic HPLC analysis. Once the extraction is complete, perform identification and quantification of the compounds via HPLC-UV or, ideally, HPLC-MS as described in refs. ${ }^{19,22}$.

(vii) SCFA analysis. To analyze SCFA, centrifuge samples $(16,000 \mathrm{~g}, 2 \mathrm{~min})$ and filter through a $0.22 \mu \mathrm{m}$ nylon filter. Right after, SCFA can be identified by HPLC-UV or HPLC-RI as described in refs. ${ }^{17,18}$. 
(C) Shotgun metagenomics and 16S rRNA analysis (for 50 samples, 1 week to extract and amplify DNA, 1 week for sequencing and analysis; shotgun metagenomics bioinformatic analysis will take longer)

(i) Extract DNA following the instructions of the extraction kit company.

(ii) Amplify DNA via PCR, as described in ref. ${ }^{18}$, for example.

(iii) Pool DNA and sequence following the instructions of the sequencing machine manufacturer.

(iv) For $16 \mathrm{~S}$ rRNA analysis, assign DNA reads to specific taxonomies via different bioinformatic tools, as described in refs. ${ }^{17-21}$. For shotgun metagenomics, sequenced gene fragments must be assembled into complete genomes before taxonomy annotation, which requires further bioinformatic processing as described in ref. ${ }^{60}$.

\section{Troubleshooting}

Troubleshooting advice can be found in Table 4 .

\begin{tabular}{|c|c|c|c|}
\hline Step & Problem & Possible reason & Possible solution \\
\hline 3 & $\begin{array}{l}\text { Solid residue from in vitro digestion is } \\
\text { often wet, viscous and hard to grab, } \\
\text { even after centrifugation. This can } \\
\text { result in taking a } \\
\text { nonhomogeneous sample }\end{array}$ & $\begin{array}{l}\text { During in vitro digestion, substrate } \\
\text { sample's structure is broken down and } \\
\text { it incorporates water from the medium }\end{array}$ & $\begin{array}{l}\text { It is essential to carefully homogenize the solid } \\
\text { residue from the in vitro digestion before adding } \\
\text { it to the in vitro fermentation tube. This will } \\
\text { ensure a homogeneous and representative } \\
\text { sample. Homogenization can be done with a } \\
\text { stainless-steel sterile spatula }\end{array}$ \\
\hline 7 & $\begin{array}{l}\text { Difficulties pipetting due to large } \\
\text { particles from fecal material that clog } \\
\text { the tips }\end{array}$ & $\begin{array}{l}\text { Due to the slow centrifugation in Step } \\
6(550 \mathrm{~g}) \text {, some large particles from } \\
\text { the fecal material could resuspend } \\
\text { eventually if pipetting and adding the } \\
\text { inoculum to the different fermentation } \\
\text { tubes takes too long }\end{array}$ & $\begin{array}{l}\text { We recommend that, immediately after } \\
\text { centrifuging, the inoculum be moved with care } \\
\text { to another vessel so that large particles remain } \\
\text { in the centrifuged tube }\end{array}$ \\
\hline 9 & $\begin{array}{l}\text { Fermentation tubes break during the } \\
\text { process }\end{array}$ & $\begin{array}{l}\text { Due to gas accumulation during } \\
\text { fermentation }\end{array}$ & $\begin{array}{l}\text { We recommend using } 50 \mathrm{~mL} \text { tubes rather than } \\
15 \mathrm{~mL} \text { tubes so that there is more space for the } \\
\text { gas, avoiding spilling }\end{array}$ \\
\hline
\end{tabular}

\section{Timing (estimated for 50 fermentation tubes)}

Steps 2 and 3, reagent preparation and autoclaving material: $2-3 \mathrm{~h}$

Steps 4 and 5, weighing the samples into fermentation tubes: highly dependent on the number of samples, for 50 samples, $1.5-2 \mathrm{~h}$

Step 6, preparation of the fecal slurry (inoculum): $1 \mathrm{~h}$ for 50 samples

Steps 7 and 8, adding components to fermentation tubes: $0.5 \mathrm{~h}$ for 50 samples

Step 9, incubation: $20 \mathrm{~h}$

Step 10, cooling, sampling and storage: $15 \mathrm{~min}$ for cooling, $1 \mathrm{~h}$ for three aliquots per sample

Step 11, sample processing:

- Option A, substrate degradation: $4 \mathrm{~h}$ if using a stove, $45 \mathrm{~h}$ if using lyophilization

- Option B, metabolite analysis: for phenolic analysis, 30-32 h for extraction and $48 \mathrm{~h}$ for identification and quantification; for SCFA, $4 \mathrm{~h}$ for extraction and $24 \mathrm{~h}$ for identification and quantification

- Option C, shotgun metagenomics and 16S RNA analysis: 2 weeks (highly dependent on laboratory resources, specifically on whether the laboratory has an automated DNA extraction system, which would reduce the timing by $\sim 1$ week)

\section{Anticipated results}

\section{Substrate degradation}

In vitro fermentation can be used to study microbial degradation of a substrate of interest ${ }^{16}$. These data could provide information about microbial capabilities to use specific foodstuffs. In a previous 
Table 5 | Fermentability of different foods

\begin{tabular}{|c|c|c|c|c|c|c|}
\hline & \multirow[b]{2}{*}{$\begin{array}{l}\text { Initial } \\
\text { amount, g }\end{array}$} & \multicolumn{2}{|c|}{ In vitro digestion } & \multicolumn{2}{|c|}{ In vitro fermentation } & \multirow[b]{2}{*}{$\begin{array}{l}\text { Final solid } \\
\text { residue, }{ }^{\mathrm{a}}\end{array}$} \\
\hline & & Digested $\%^{\mathrm{a}}$ & $\begin{array}{l}\text { Non- } \\
\text { digested } \\
\%^{\mathrm{a}}\end{array}$ & Fermented $\%^{\mathrm{a}}$ & Nonfermented $\%^{a}$ & \\
\hline $\begin{array}{l}\text { Whole- } \\
\text { grain bread }\end{array}$ & 5.00 & 55.07 & 44.93 & 65.54 & 34.46 & 15.48 \\
\hline Lentils & 5.00 & 60.48 & 39.52 & 55.32 & 44.68 & 17.66 \\
\hline Orange & 5.00 & 36.83 & 63.17 & 65.05 & 34.95 & 22.08 \\
\hline Tomato & 5.00 & 59.74 & 40.26 & 72.46 & 27.54 & 11.09 \\
\hline Yogurt & 5.00 & 86.34 & 13.66 & 76.39 & 23.61 & 3.23 \\
\hline Peanuts & 5.00 & 4.19 & 95.81 & 26.88 & 73.12 & 70.06 \\
\hline
\end{tabular}

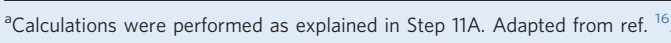

experiment, we studied the fermentability of several foodstuffs (Table 5, adapted from ref. ${ }^{16}$ ). Reproducibility was also tested, showing an interday variation coefficient of $5.18 \%$ and an intraday variation coefficient of $5.26 \%$.

\section{Study of phytochemicals and microbial metabolic pathways}

We studied phenolic compounds released from green and roasted coffee ${ }^{18}$ and melanoidins ${ }^{21}$ after microbial fermentation as well as their metabolites. Coffee brew fermentation yielded much higher amounts of $4^{\prime}$-hydroxy-3'-methoxycinnamic acid (ferulic acid), 4-hydroxybenzoic acid and $4^{\prime}$ hydroxyphenyl-acetic acid than found in coffee brew before fermentation, all related to chlorogenic acid microbial degradation ${ }^{18}$. In another study, the fermentation of melanoidins yielded high concentrations of different benzoic acids $\left(\mathrm{C}_{6}-\mathrm{C}_{1}\right)$, related to phenolic degradation ${ }^{25}$. For fermented coffee and chocolate melanoidins, high concentrations of benzene-1,2,3-triol (pyrogallol) were found. The latter is a flavan-3-ol metabolite ${ }^{24}$, and flavan-3-ol is known to be incorporated into the structure of coffee and chocolate melanoidins ${ }^{61}$.

The data obtained in the previously mentioned experiments is not only useful to understand how different molecules are metabolized by gut microbes, but also to be able to predict the metabolic behavior of such bacteria in different contexts by building metabolic networks and constraint-based modeling ${ }^{46,62}$. In a previous study, this approach was applied to unravel the metabolic changes that occur during the first year of life in the gut microbiota of a cohort of Spanish infants ${ }^{35}$. This study showed how the introduction of solid food generated a different signature of metabolites released by gut microbes, which was validated through experimental data.

\section{Gut microbial functionality}

Gut microbial functionality is most commonly measured through SCFA (mainly acetate, propionate and butyrate) production since they are the main microbial fermentation products ${ }^{1}$. According to epidemiological data, high-fiber low-fat and low-meat diets result in a higher SCFA production than those with low fiber consumption ${ }^{6,63}$. Therefore, foods with higher fiber content or added fiber should, in theory, increase SCFA production. This was demonstrated in previous batch fermentations experiments: in one of them, mannoligosaccharides increased SCFA in a dose-dependent manner ${ }^{20}$; in another one, the addition of different fibers to salami increased SCFA production, especially butyrate production $^{19}$, which agrees with data found in vivo ${ }^{6}$, and also with the results found of a human intervention where the same salami was tested ${ }^{44}$. It is expected to find higher concentrations of SCFA after fermenting fiber-rich foods, an expectation that was confirmed in a previous experiment where fermentations of pepper, bread, banana or chickpeas showed higher SCFA concentrations than with chicken ${ }^{17}$. If the experiment also involves microbial analysis (16S rRNA amplicon sequencing or shotgun metagenomics), it is expected to find some correlations with SCFA-producing bacteria $^{17}$, such as Ruminococcus or Bifidobacterium, which are major acetate producing genera; Faecalibacterium and Eubacterium, the top butyrate producers; and Roseburia or Blautia, which are the main propionate producers ${ }^{1}$. 

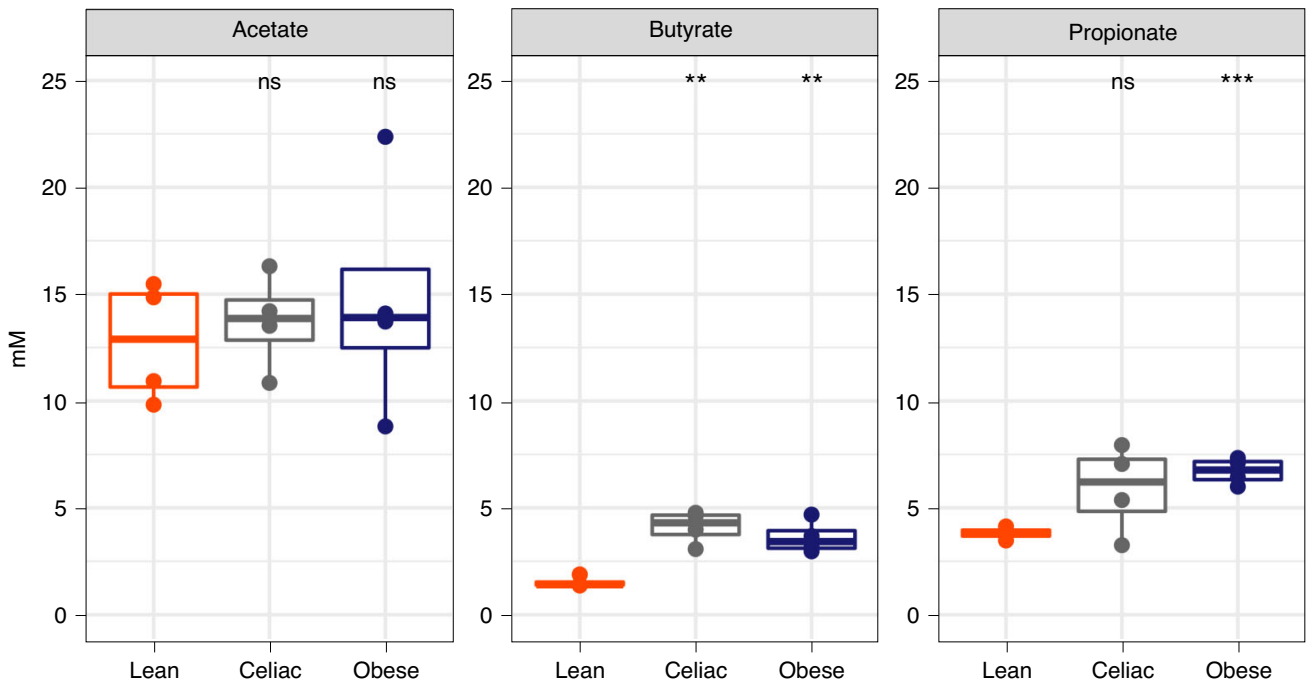

Fig. 3 | Microbial functionality affected by specific health conditions. SCFA (acetate, butyrate and propionate) production after in vitro fermentation of lentils with feces from 12 individuals: four lean, four celiac and four obese adults. Statistical differences were calculated by means of one-way ANOVA using lean as the reference group. Significance labels: ns, not significant; ${ }^{\star \star} P<0.01 ;{ }^{\star \star \star} P<0.001$.

Other fermentation metabolites-mainly fumarate, succinate and lactate-involved in crossfeeding mechanisms can also be assessed. However, they are usually found in very low concentrations since they are used by different bacteria; for example, lactate can be used to produce propionate and butyrate $^{25}$. Regardless, in certain circumstances they can be useful; for instance, lactate is abnormally high in patients with ulcerative colitis ${ }^{64}$.

Acetate, propionate and butyrate are the main metabolites from carbohydrate degradation. However, gut microbes have also important proteolytic activity, which yields different compounds such as peptides, amino acids, branched-chain fatty acids or ammonia ${ }^{25}$. Some of the protein-derived metabolites are negatively associated with host health, such as trimethylamine, ammonia or hydrogen sulfide ${ }^{4}$. According to Shankar et al. ${ }^{4}$, a typical Western diet, rich in animal products and refined cereals, is characterized by a predominance of proteolytic microbial communities, whereas populations with fiber-rich diets are characterized by bacteria responsible for carbohydrate degradation. Therefore, the measurement of both SCFA and protein metabolites could give a preliminary view of the metabolic preferences of gut microbes and indicate how the microbial functionality can be shifted depending on the substrate given.

Specific health conditions might also affect gut microbial composition and, hence, their functionality. For instance, SCFA concentration in feces of obese/overweight people and celiac patients is higher than in lean and healthy people ${ }^{63,65}$. We tested this protocol using fecal material from four lean, four celiac and four obese individuals to ferment lentils (Fig. 3). The results obtained with this protocol are in accordance with those described by Fernandes et al. ${ }^{63}$ and Nistal et al.$^{65}$, who observed how acetate, propionate and butyrate concentration were higher in obese ${ }^{63}$ and celiac ${ }^{65}$ people than in lean or healthy people. However, our results showed how differences were only statistically significant $(P<0.05)$ in the case of butyrate production between groups and in propionate production between lean and celiac people. It has been reported that the gut microbiota associated with obese people are able to scavenge higher amounts of energy from substrates and, hence, have higher production of $\mathrm{SCFA}^{63}$. In the case of celiac people, the differences between this group and lean people are not as clear. These results show how the protocol is able to reflect the SCFA production ability of the original feces. In this sense, the in vitro batch fermentation protocol described here has been successfully used in several studies to measure SCFA production ${ }^{17-21}$.

\section{Gut microbial community structure}

One of the main outcomes of in vitro fermentations is data regarding gut microbial community structure. In vitro batch fermentations are especially useful to explore how gut microbes can use different foods or molecules. 


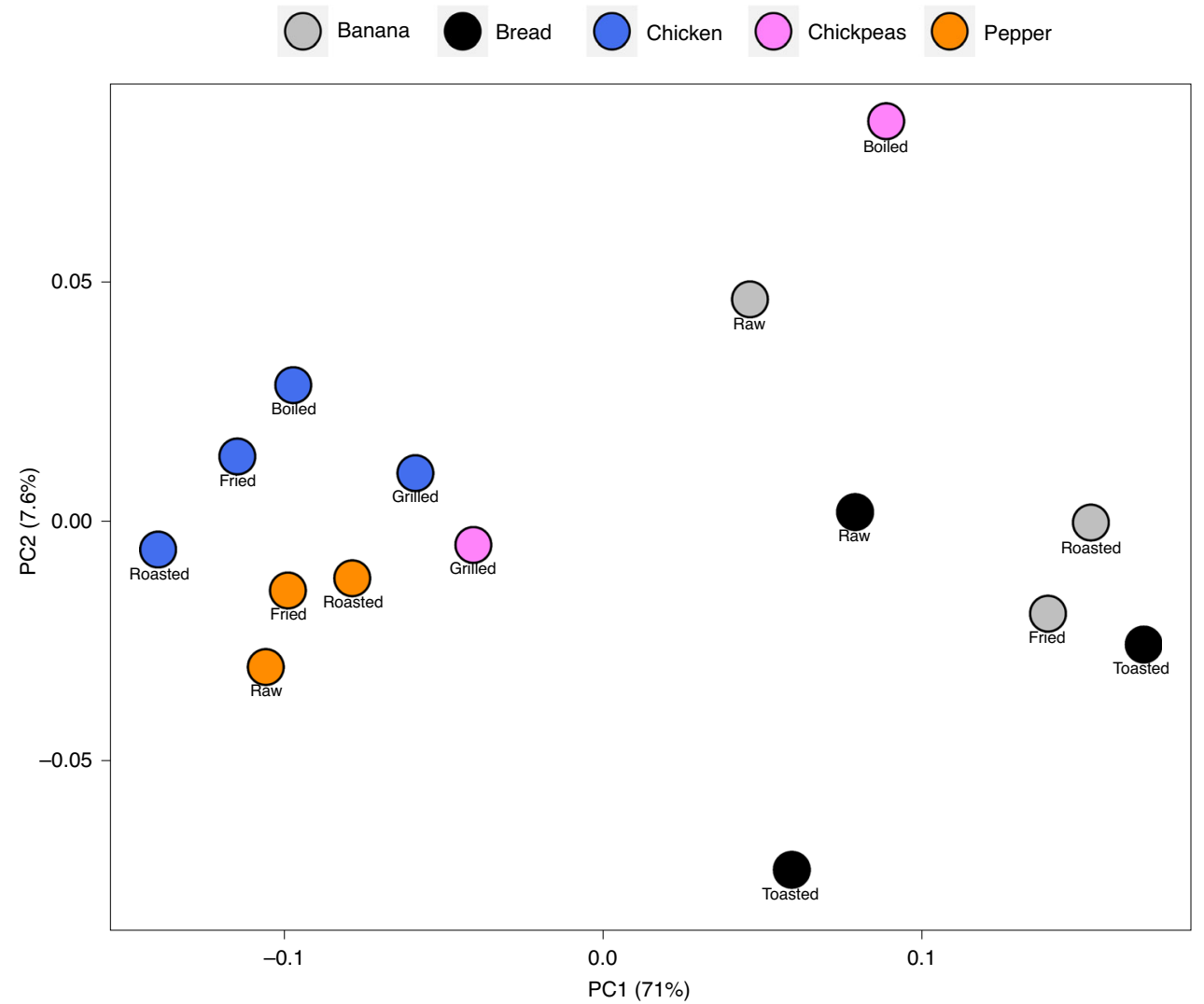

Fig. 4 | Differences in gut microbial community structure after fermenting different foods. Principal coordinates analysis of genus abundance among all 15 profiled samples ( 4 chicken, 3 pepper, 3 banana, 3 bread and 2 chickpea), as previously reported by Perez-Burillo et al. ${ }^{17}$. Phylogenetic weighted UniFrac distance was used to calculate the sample dissimilarity matrix. Samples (dots) closer together indicate similarity among those samples. Samples farther away indicate dissimilarity among those samples. Image reproduced with permission from ref. ${ }^{17}$.

Two typical datasets obtained are those in which the aim is to identify differences in microbial community structure after fermenting different foods, and those in which the aim is to study the effects of potential prebiotic agents. In a previous research project, we investigated the effect that different foods (chicken, chickpeas, pepper, bread and banana) could have on gut microbial structure $^{17}$. Through multivariable analysis, we found a clear difference between the communities exposed to the different foods (Fig. 4), with the structure of the communities given protein-rich foods closer to one another, starchy foods also closer to each other, and the fiber-rich food (pepper) separated from the rest. Interesting information regarding SCFA-producing genera was also found, which was backed by existing literature, as Ruminococcus was found in higher abundance in communities given starchy foods ${ }^{66}$, and a higher abundance of butyrate-producing genera was observed with higher-fibercontent foods ${ }^{6}$. We also investigated the potential use of food melanoidins as prebiotic agents ${ }^{21}$. In this regard, we found that biscuit melanoidins stimulated the growth of Faecalibacterium (a butyrate producer), whereas others (such as bread crust melanoidins) stimulated the growth of Bifidobacterium.

\section{Reporting Summary}

Further information on research design is available in the Nature Research Reporting Summary linked to this article.

\section{Data availability}

The data shown in Fig. 4 are available from the supporting primary research paper previously published by Pérez-Burillo et al. ${ }^{17}$. The data presented in Figs. 2 and 3 were generated for this protocol. Source data are provided with this paper. 
1. Flint, H. J., Duncan, S. H., Scott, K. P. \& Louis, P. Links between diet, gut microbiota composition and gut metabolism. Proc. Nutr. Soc. 74, 13-22 (2015).

2. Salazar, N. et al. Exopolysaccharides produced by Bifidobacterium longum IPLA E44 and Bifidobacterium animalis subsp. lactis IPLA R1 modify the composition and metabolic activity of human faecal microbiota in pH-controlled batch cultures. Int. J. Food Microbiol. 135, 260-267 (2009).

3. Power, S. E., O’Toole, P. W., Stanton, C., Ross, R. P. \& Fitzgerald, G. F. Intestinal microbiota, diet and health. Br. J. Nutr. 111, 387-402 (2014).

4. Shankar, V. et al. Differences in gut metabolites and microbial composition and functions between Egyptian and U.S. children are consistent with their diets. mSystems 2, e00169-16 (2017).

5. David, L. A. et al. Diet rapidly and reproducibly alters the human gut microbiome. Nature 505, 559-563 (2013).

6. De Filippo, C. et al. Impact of diet in shaping gut microbiota revealed by a comparative study in children from Europe and rural Africa. Proc. Natl Acad. Sci. USA 107, 14691-14696 (2010).

7. Venema, K. \& van den Abbeele, P. Experimental models of the gut microbiome. Best Pract. Res. Clin. Gastroenterol. 27, 115-126 (2013).

8. Molly, K., Vande Woestyne, M. \& Verstraete, W. Development of a 5-step multi-chamber reactor as a simulation of the human intestinal microbial ecosystem. Appl. Microbiol. Biotechnol. 39, 254-258 (1993).

9. Agans, R. et al. Dietary fatty acids sustain the growth of the human gut microbiota. Appl. Environ. Microbiol. 84, e01525-18 (2018).

10. Wiese, M. et al. CoMiniGut-a small volume in vitro colon model for the screening of gut microbial fermentation processes. PeerJ 6, e4268 (2018).

11. Ludwig, I. A., Paz de Peña, M., Concepción, C. \& Alan, C. Catabolism of coffee chlorogenic acids by human colonic microbiota: colonic catabolism of coffee chlorogenic acids. BioFactors 39, 623-632 (2013).

12. Coles, L. T., Moughan, P. J. \& Darragh, A. J. In vitro digestion and fermentation methods, including gas production techniques, as applied to nutritive evaluation of foods in the hindgut of humans and other simple-stomached animals. Anim. Feed Sci. Technol. 123-124, 421-444 (2005).

13. Wang, M. et al. In vitro colonic fermentation of dietary fibers: fermentation rate, short-chain fatty acid production and changes in microbiota. Trends Food Sci. Technol. 88, 1-9 (2019).

14. Mould, F. L., Morgan, R., Kliem, K. E. \& Krystallidou, E. A review and simplification of the in vitro incubation medium. Anim. Feed Sci. Technol. 123-124, 155-172 (2005).

15. Brodkorb, A. et al. INFOGEST static in vitro simulation of gastrointestinal food digestion. Nat. Protoc. 14, 991-1014 (2019).

16. Pérez-Burillo, S., Rufián-Henares, J. A. \& Pastoriza, S. Towards an improved global antioxidant response method $(\mathrm{GAR}+)$ : physiological-resembling in vitro digestion-fermentation method. Food Chem 239, 1253-1262 (2018).

17. Pérez-Burillo, S. et al. Effect of food thermal processing on the composition of the gut microbiota. J. Agric. Food Chem. 66, 11500-11509 (2018).

18. Pérez-Burillo, S. et al. Effect of in vitro digestion-fermentation on green and roasted coffee bioactivity: the role of the gut microbiota. Food Chem. 279, 252-259 (2019).

19. Pérez-Burillo, S. et al. Potential probiotic salami with dietary fiber modulates antioxidant capacity, short chain fatty acid production and gut microbiota community structure. LWT 105, 355-362 (2019).

20. Pérez-Burillo, S. et al. Spent coffee grounds extract, rich in mannooligosaccharides, promotes a healthier gut microbial community in a dose-dependent manner. J. Agric. Food Chem. 67, 2500-2509 (2019).

21. Pérez-Burillo, S., Rajakaruna, S., Pastoriza, S., Paliy, O. \& Rufián-Henares, J. A. Bioactivity of food melanoidins is mediated by gut microbiota. Food Chem. 316, 126309 (2020).

22. Rocchetti, G. et al. Transformation of polyphenols found in pigmented gluten-free flours during in vitro large intestinal fermentation. Food Chem. 298, 125068 (2019).

23. Jin, J. B. et al. Supplementation with Chlorella vulgaris, Chlorella protothecoides, and Schizochytrium sp. increases propionate-producing bacteria in in vitro human gut fermentation. J. Sci. Food Agric. 100, 2938-2945 (2020).

24. Selma, M. V., Espín, J. C. \& Tomás-Barberán, F. A. Interaction between phenolics and gut microbiota: role in human health. J. Agric. Food Chem. 57, 6485-6501 (2009).

25. Rowland, I. et al. Gut microbiota functions: metabolism of nutrients and other food components. Eur. J. Nutr. 57, 1-24 (2018).

26. Tomas-Barberan, F. et al. In vitro transformation of chlorogenic acid by human gut microbiota. Mol. Nutr. Food Res. 58, 1122-1131 (2014).

27. Saura-Calixto, F. et al. Proanthocyanidin metabolites associated with dietary fibre from in vitro colonic fermentation and proanthocyanidin metabolites in human plasma. Mol. Nutr. Food Res. 54, 939-946 (2010).

28. Roowi, S. et al. Green tea flavan-3-ols: colonic degradation and urinary excretion of catabolites by humans. $J$. Agric. Food Chem. 58, 1296-1304 (2010).

29. Jaganath, I. B., Mullen, W., Lean, M. E. J., Edwards, C. A. \& Crozier, A. In vitro catabolism of rutin by human fecal bacteria and the antioxidant capacity of its catabolites. Free Radic. Biol. Med. 47, 1180-1189 (2009).

30. Serra, A. et al. Metabolic pathways of the colonic metabolism of flavonoids (flavonols, flavones and flavanones) and phenolic acids. Food Chem. 130, 383-393 (2012). 
31. Pinta, M. N. et al. In vitro gut metabolism of [U-13C]-quinic acid, the other hydrolysis product of chlorogenic acid. Mol. Nutr. Food Res. 62, 1800396 (2018).

32. Hidalgo, M. et al. Metabolism of anthocyanins by human gut microflora and their influence on gut bacterial growth. J. Agric. Food Chem. 60, 3882-3890 (2012).

33. Marín, L., Miguélez, E. M., Villar, C. J. \& Lombó, F. Bioavailability of dietary polyphenols and gut microbiota metabolism: antimicrobial properties. Biomed. Res. Int. https://doi.org/10.1155/2015/905215 (2015).

34. Stevens, J. F. \& Maier, C. S. The chemistry of gut microbial metabolism of polyphenols. Phytochem. Rev. 15, 425-444 (2016).

35. Fuertes, Á. et al. Adaptation of the human gut microbiota metabolic network during the first year after birth. Front. Microbiol. 10, 848 (2019).

36. Ríos-Covián, D. et al. Intestinal short chain fatty acids and their link with diet and human health. Front. Microbiol. 7, 185 (2016).

37. Shen, Q., Chen, Y. A. \& Tuohy, K. M. A comparative in vitro investigation into the effects of cooked meats on the human faecal microbiota. Anaerobe 16, 572-577 (2010).

38. Poelaert, C. et al. Cooking has variable effects on the fermentability in the large intestine of the fraction of meats, grain legumes, and insects that is resistant to digestion in the small intestine in an in vitro model of the pig's gastrointestinal tract. J. Agric. Food Chem. 65, 435-444 (2017).

39. Lee, D. K. et al. The combination of mixed lactic acid bacteria and dietary fiber lowers serum cholesterol levels and fecal harmful enzyme activities in rats. Arch. Pharm. Res. 34, 23-29 (2011).

40. Molska, M. \& Regula, J. Potential mechanisms of probiotics action in the prevention and treatment of colorectal cancer. Nutrients 11, 2453 (2019).

41. Pham, V. T. \& Mohajeri, M. H. The application of in vitro human intestinal models on the screening and development of pre- and probiotics. Benef. Microbes 9, 725-742 (2018).

42. $\mathrm{Gu}, \mathrm{F}$. et al. In vitro fermentation behavior of isomalto/malto-polysaccharides using human fecal inoculum indicates prebiotic potential. Mol. Nutr. Food Res. 62, e1800232 (2018).

43. Fehlbaum, S. et al. In vitro fermentation of selected prebiotics and their effects on the composition and activity of the adult gut microbiota. Int. J. Mol. Sci. 19, 3097 (2018).

44. Pérez-Burillo, S. et al. Potential probiotic salami with dietary fiber modulates metabolism and gut microbiota in a human intervention study. J. Funct. Foods 66, 103790 (2020).

45. Kolodziejczyk, A. A., Zheng, D. \& Elinav, E. Diet-microbiota interactions and personalized nutrition. Nat. Rev. Microbiol. 17, 742-753 (2019).

46. Magnúsdóttir, S. et al. Generation of genome-scale metabolic reconstructions for 773 members of the human gut microbiota. Nat. Biotechnol. 35, 81-89 (2017).

47. Coyte, K. Z. \& Rakoff-Nahoum, S. Understanding competition and cooperation within the mammalian gut microbiome. Curr. Biol. 29, R538-R544 (2019).

48. Payne, A. N., Zihler, A., Chassard, C. \& Lacroix, C. Advances and perspectives in in vitro human gut fermentation modeling. Trends Biotechnol. 30, 17-25 (2012).

49. Cohen, S. M. Human relevance of animal carcinogenicity studies. Regul. Toxicol. Pharmacol. 21, 75-80 (1995). discussion 81-86.

50. Eckburg, P. B. et al. Diversity of the human intestinal microbial flora. Science 308, 1635-1638 (2005).

51. Ley, R. E. et al. Obesity alters gut microbial ecology. Proc. Natl Acad. Sci. USA 102, 11070-11075 (2005).

52. Belkaid, Y. \& Hand, T. W. Role of the microbiota in immunity and inflammation. Cell 157, 121-141 (2014).

53. Shankar, V. et al. The networks of human gut microbe-metabolite associations are different between health and irritable bowel syndrome. ISME J. 9, 1899-1903 (2015).

54. Aguirre, M. \& Venema, K. Challenges in simulating the human gut for understanding the role of the microbiota in obesity. Benef. Microbes 8, 31-53 (2017).

55. Gratton, J. et al. Optimized sample handling strategy for metabolic profiling of human feces. Anal. Chem. 88, 4661-4668 (2016).

56. Gorzelak, M. A. et al. Methods for improving human gut microbiome data by reducing variability through sample processing and storage of stool. PLoS ONE 10, e0134802 (2015).

57. Long, W. et al. Differential responses of gut microbiota to the same prebiotic formula in oligotrophic and eutrophic batch fermentation systems. Sci. Rep. 5, 1-11 (2015).

58. Paliy, O. \& Shankar, V. Application of multivariate statistical techniques in microbial ecology. Mol. Ecol. 25, 1032-1057 (2016).

59. Pérez-Burillo, S., Rufián-Henares, J. A. \& Pastoriza, S. Effect of home cooking on the antioxidant capacity of vegetables: relationship with Maillard reaction indicators. Food Res. Int. 121, 514-523 (2019).

60. Quince, C., Walker, A. W., Simpson, J. T., Loman, N. J. \& Segata, N. Shotgun metagenomics, from sampling to analysis. Nat. Biotechnol. 35, 833-844 (2017).

61. Morales, F. J., Somoza, V. \& Fogliano, V. Physiological relevance of dietary melanoidins. Amino Acids 42, 1097-1109 (2012).

62. Bauer, E. \& Thiele, I. From network analysis to functional metabolic modeling of the human gut microbiota. mSystems 3, 00209-00217 (2018).

63. Fernandes, J., Su, W., Rahat-Rozenbloom, S., Wolever, T. M. S. \& Comelli, E. M. Adiposity, gut microbiota and faecal short chain fatty acids are linked in adult humans. Nutr. Diabetes 4, e121-e121 (2014).

64. Bjerrum, J. T. et al. Metabonomics of human fecal extracts characterize ulcerative colitis, Crohn's disease and healthy individuals. Metabolomics 11, 122-133 (2015). 
65. Nistal, E. et al. Differences in faecal bacteria populations and faecal bacteria metabolism in healthy adults and celiac disease patients. Biochimie 94, 1724-1729 (2012).

66. Ze, X., Duncan, S. H., Louis, P. \& Flint, H. J. Ruminococcus bromii is a keystone species for the degradation of resistant starch in the human colon. ISME J 6, 1535-1543 (2012).

67. Zhou, L., Xie, M., Yang, F. \& Liu, J. Antioxidant activity of high purity blueberry anthocyanins and the effects on human intestinal microbiota. LWT 117, 108621 (2020).

68. Gonçalves, G. A. et al. Effects of in vitro gastrointestinal digestion and colonic fermentation on a rosemary (Rosmarinus officinalis L) extract rich in rosmarinic acid. Food Chem. 271, 393-400 (2019).

69. Chen, Y., Chang, S. K. C., Zhang, Y., Hsu, C.-Y. \& Nannapaneni, R. Gut microbiota and short chain fatty acid composition as affected by legume type and processing methods as assessed by simulated in vitro digestion assays. Food Chem. 312, 126040 (2020).

70. del Hierro, J. N. et al. In vitro colonic fermentation of saponin-rich extracts from quinoa, lentil, and fenugreek. effect on sapogenins yield and human gut microbiota. J. Agric. Food Chem. 68, 106-116 (2020).

71. Zhang, X. et al. Phytochemical profile, bioactivity, and prebiotic potential of bound phenolics released from rice bran dietary fiber during in vitro gastrointestinal digestion and colonic fermentation. J. Agric. Food Chem. 67, 12796-12805 (2019).

72. Wang, M. et al. Purified fraction of polysaccharides from Fuzhuan brick tea modulates the composition and metabolism of gut microbiota in anaerobic fermentation in vitro. Int. J. Biol. Macromol. 140, 858-870 (2019).

73. Rui, Y. et al. Simulated digestion and fermentation in vitro by human gut microbiota of intra- and extra-cellular polysaccharides from Aspergillus cristatus. LWT 116, 108508 (2019).

74. Chen, L. et al. Simulated digestion and fermentation in vitro by human gut microbiota of polysaccharides from Helicteres angustifolia L. Int. J. Biol. Macromol. 141, 1065-1071 (2019).

75. Huang, F. et al. Structural characterization and in vitro gastrointestinal digestion and fermentation of litchi polysaccharide. Int. J. Biol. Macromol. 140, 965-972 (2019).

\section{Acknowledgements}

This study was supported by the EU project Stance4Health (contract no. 816303) and by the Plan propio de Investigación y Transferencia of the University of Granada under the program 'Intensificación de la Investigación, modalidad B' to J.A.R.-H.

\section{Author contributions}

S.P.-B., S.M., and J.A.R.-H. wrote the manuscript. B.N.-P., A.J.V.-M., D.H.-N., A.L.-M. and S.P. contributed to the writing of the manuscript. S.P.-B., S.M., B.N.-P., A.J.V.-M., D.H.-N. and A.L.-M. contributed to formal analysis and investigation; S.P.-B. developed the methodology. S.P. and J.A.R.-H. supervised the work. J.A.R.-H. obtained funding and coordinates the EU project Stance4Health.

\section{Competing interests}

The authors declare no competing interests.

\section{Additional information}

Supplementary information The online version contains supplementary material available at https://doi.org/10.1038/s41596-021-00537-X. Correspondence and requests for materials should be addressed to J.A.R.-H.

Peer review information Nature Protocols thanks Francisco Tomás-Barberán and the other, anonymous reviewer(s) for their contribution to the peer review of this work.

Reprints and permissions information is available at www.nature.com/reprints.

Publisher's note Springer Nature remains neutral with regard to jurisdictional claims in published maps and institutional affiliations.

Received: 28 September 2020; Accepted: 18 March 2021;

Published online: 4 June 2021

\section{Related links}

Key references using this protocol

Pérez-Burillo, S. et al. Food Chem. 239, 1253-1262 (2018): https://doi.org/10.1016/j.foodchem.2017.07.024

Pérez-Burillo, S. et al. Food Chem. 279, 252-259 (2019): https://doi.org/10.1016/j.foodchem.2018.11.137

Pérez-Burillo, S., et al. Food Chem. 316, 126309 (2020): https://doi.org/10.1016/j.foodchem.2020.126309 


\section{Reporting Summary}

Nature Research wishes to improve the reproducibility of the work that we publish. This form provides structure for consistency and transparency in reporting. For further information on Nature Research policies, see our Editorial Policies and the Editorial Policy Checklist.

Please do not complete any field with "not applicable" or $\mathrm{n} / \mathrm{a}$. Refer to the help text for what text to use if an item is not relevant to your study. For final submission: please carefully check your responses for accuracy; you will not be able to make changes later.

\section{Statistics}

For all statistical analyses, confirm that the following items are present in the figure legend, table legend, main text, or Methods section.

$\mathrm{n} / \mathrm{a} \mid$ Confirmed

X The exact sample size $(n)$ for each experimental group/condition, given as a discrete number and unit of measurement

X A statement on whether measurements were taken from distinct samples or whether the same sample was measured repeatedly

$X$ The statistical test(s) used AND whether they are one- or two-sided

Only common tests should be described solely by name; describe more complex techniques in the Methods section.

$X$ A description of all covariates tested

X description of any assumptions or corrections, such as tests of normality and adjustment for multiple comparisons

$\mathrm{X}$ Aull description of the statistical parameters including central tendency (e.g. means) or other basic estimates (e.g. regression coefficient)

AND variation (e.g. standard deviation) or associated estimates of uncertainty (e.g. confidence intervals)

For null hypothesis testing, the test statistic (e.g. $F, t, r$ ) with confidence intervals, effect sizes, degrees of freedom and $P$ value noted

Give $P$ values as exact values whenever suitable.

For Bayesian analysis, information on the choice of priors and Markov chain Monte Carlo settings

For hierarchical and complex designs, identification of the appropriate level for tests and full reporting of outcomes

Estimates of effect sizes (e.g. Cohen's $d$, Pearson's $r$ ), indicating how they were calculated

Our web collection on statistics for biologists contains articles on many of the points above.

\section{Software and code}

Policy information about availability of computer code

Data collection Provide a description of all commercial, open source and custom code used to collect the data in this study, specifying the version used oR state that no software was used.

Data analysis

Provide a description of all commercial, open source and custom code used to analyse the data in this study, specifying the version used OR state that no software was used.

For manuscripts utilizing custom algorithms or software that are central to the research but not yet described in published literature, software must be made available to editors and reviewers. We strongly encourage code deposition in a community repository (e.g. GitHub). See the Nature Research guidelines for submitting code \& software for further information.

\section{Data}

Policy information about availability of data

All manuscripts must include a data availability statement. This statement should provide the following information, where applicable:

- Accession codes, unique identifiers, or web links for publicly available datasets

- A list of figures that have associated raw data

- A description of any restrictions on data availability

The data shown in Figure 4 is available from the supporting primary research paper previously published by Perez-Burillo et al.17.

- The data presented in Figs. $2 \& 3$ were generated for this protocol.

- The source data underlying Figs. $2 \& 3$ are provided as Source Data files with this protocol. 
Field-specific reporting

Please select the one below that is the best fit for your research. If you are not sure, read the appropriate sections before making your selection.

\Life sciences

Behavioural \& social sciences

Ecological, evolutionary \& environmental sciences

\section{Life sciences study design}

All studies must disclose on these points even when the disclosure is negative.

Sample size

Not needed for a protocol paper

Data exclusions

Not needed for a protocol paper

Replication

Not needed for a protocol paper

Randomization

Not needed for a protocol paper

Blinding

Not needed for a protocol paper

\section{Reporting for specific materials, systems and methods}

We require information from authors about some types of materials, experimental systems and methods used in many studies. Here, indicate whether each material, system or method listed is relevant to your study. If you are not sure if a list item applies to your research, read the appropriate section before selecting a response.

Materials \& experimental systems Methods

$\mathrm{n} / \mathrm{a}$ Involved in the study

X $\square$ Antibodies $\mathrm{n} / \mathrm{a}$ Involved in the study

$\searrow \square$ Eukaryotic cell lines

X $\square$ ChIP-seq

Х $\square$ Palaeontology and archaeology

X $\square$ Flow cytometry

$\searrow \square$ Animals and other organisms

X $\square$ MRI-based neuroimaging

$\square \square$ Human research participants

$\searrow \square$ Clinical data

$\searrow \square$ Dual use research of concern

\section{Human research participants}

Policy information about studies involving human research participants

Population characteristics

Not needed for a protocol paper

Recruitment

Describe how participants were recruited. Outline any potential self-selection bias or other blases that may be present and how these are likeiy to impact results.

Ethics oversight

Not needed for a protocol paper

Note that full information on the approval of the study protocol must also be provided in the manuscript. 